\title{
A physical mechanism producing suprathermal populations and initiating substorms in the Earth's magnetotail
}

\author{
D. V. Sarafopoulos \\ Department of Electrical and Computer Engineering, Democritus University of Thrace, Xanthi, Greece
}

Received: 1 March 2007 - Revised: 17 March 2008 - Accepted: 16 April 2008 - Published: 11 June 2008

\begin{abstract}
We suggest a candidate physical mechanism, combining there dimensional structure and temporal development, which is potentially able to produce suprathermal populations and cross-tail current disruptions in the Earth's plasma sheet. At the core of the proposed process is the "akis" structure; in a thin current sheet (TCS) the stretched (tail-like) magnetic field lines locally terminate into a sharp tip around the tail midplane. At this sharp tip of the TCS, ions become non-adiabatic, while a percentage of electrons are accumulated and trapped: The strong and transient electrostatic electric fields established along the magnetic field lines produce suprathermal populations. In parallel, the tip structure is associated with field aligned and mutually attracted parallel filamentary currents which progressively become more intense and inevitably the structure collapses, and so does the local TCS. The mechanism is observationally based on elementary, almost autonomous and spatiotemporal entities that correspond each to a local thinning/dipolarization pair having duration of $\sim 1 \mathrm{~min}$. Energetic proton and electron populations do not occur simultaneously, and we infer that they are separately accelerated at local thinnings and dipolarizations, respectively. In one example energetic particles are accelerated without any $d B / d t$ variation and before the substorm expansion phase onset. A particular effort is undertaken demonstrating that the proposed acceleration mechanism may explain the plasma sheet ratio $T_{i} / T_{e} \approx 7$. All our inferences are checked by the highest resolution datasets obtained by the Geotail Energetic Particles and Ion Composition (EPIC) instrument. The energetic particles are used as the best diagnostics for the accelerating source. Near Earth $\left(X \approx 10 R_{E}\right.$ ) selected events support our basic concept. The proposed mechanism seems to reveal a fundamental building block of the substorm phenomenon and may be the basic process/structure, which is now missing, that might help ex-
\end{abstract}

Correspondence to: D. V. Sarafopoulos

(sarafo@ee.duth.gr) plain the persistent, outstanding deficiencies in our physical description of magnetospheric substorms. The mechanism is tested, checked, and found consistent with substorm associated observations performed $\sim 30$ and $60 R_{E}$ away from Earth.

Keywords. Magnetospheric physics (Magnetospheric configuration and dynamics; Plasma sheet) - Space plasma physics (Charged particle motion and acceleration)

\section{Introduction}

At the start of this paper we want to state that our primary intent and motivation in this work was and remains neither to be involved in a longstanding contradiction justifying one side, nor to resolve the contradiction itself being the "where, how and when" a substorm initiates. This work came about, rather, as an effort to check whether a well known fundamental concept was at work in the magnetotail dynamics. It is known from electrostatics that the surface charge densities are greatest at sharp tips of conductor, and so the local electric field strength actually produce an increased charge accumulation at the sharp tip part (i.e. the very low curvature region) of an already stretched magnetic field line? Might a negatively charged sharp tip be the ultimate substorm triggering mechanism? Is there a capability for a sharp tip to produce suprathermal populations in the Earth's magnetosphere?

In the Earth's magnetotail suprathermal particles are always present independent of geomagnetic activity (Christon et al., 1989, 1991). Their fluxes depend on the temperature of the thermal population, being high when the temperature is high, and low when the temperature is low, that is, low and high energy components act as complementary parts of a single magnetospheric population. However, their origin, although presumably related to some form of scattering and

Published by Copernicus Publications on behalf of the European Geosciences Union. 
energization in the turbulent fields of the plasma sheet, appears to be unresolved at present (Cowley, 1991; Heikkila, 2007).

We postulate that in the Earth's magnetotail an instability must exist as the direct cause of substorms and that expansion waves, cross-tail current disruption, reconnection, particle energization and other dynamic phenomena may well be the nonlinear effect, but not the cause, of the growth of an unstable mode. There have been several suggestions for such instability like the cross-field current instability (Lui et al., 1991), the ballooning instability model (Roux et al., 1991), and the tearing instability responsible for the onset of reconnection (Schindler, 1974).

In this work, we propose a candidate physical mechanism that may potentially trigger substorms, producing suprathermal populations. In parallel, we are interested in testing the mechanism using available energetic particle datasets. We require high time-resolution measurements for protons and electrons, in order to trace possible transient accelerating electric fields. In particular, this work is mainly based on the highest resolution data obtained by the Geotail/EPIC instrument. Finally, we infer that our main suggestion is supported observationally as no serious conflict is found. However, these longstanding, multi-dimensional and open problems need further work to be definitely resolved. We believe that this work indicates the right direction and gives a few decisive answers.

We shall elaborate our mechanism throughout this work: A close coupling and interaction between the in situ measurements and our basic concept is our chief concern. However, although many aspects of the work will be clarified in the course of the paper, a preliminary description of our concept is of primary importance, being at the same time somewhat arbitrary for the reader. We are especially focused on local (likely less than $1 R_{E}$ ), short-lived (less than $1 \mathrm{~min}$ ), and usually successive reconfigurations of the magnetic field accompanied by high energetic particle populations. Fundamental knowledge concerning the substorm phenomenon can be found in reviews like those of Lui (1996), Baker et al. (1996), Sergeev et al. (1996), Friedel et al. (1996), Lyons (1996), Birn and Schindler (1996), and Rostoker (1996). Briefly, the basic description of the proposed mechanism is as follows:

1. During the substorm growth phase the magnetotail is stretched to a tail-like topology: A thin current sheet (TCS) is developed.

2. The magnetic field lines locally form a "sharp tip", or an "akis" structure around the tail midplane. "Akis" (i.e. $\dot{\alpha} \kappa i \zeta$ ) is an ancient Greek word giving the best description for stretched lines terminating into a point.

3. At the sharp tip the ions become non-adiabatic, and the involved magnetic field lines are negatively charged.

4. A test electron particle close to the tip initially feels an outward force greater than an inward. Hence, elec- trons, with smaller gyroradii, are able to follow the field more closely and are accumulated and trapped in the tip structure, which may eventually accommodate extremely high charge densities.

5. Strong electrostatic electric fields along the magnetic field lines are established producing suprathermal ions.

6. The tip inevitably collapses, since it progressively becomes sharper driving higher current densities. These currents, along the magnetic field lines, are mutually attracted like parallel current filaments.

7. The collapsing tip structure is associated with the highest local negative charge density, which can produce a strong-transient and earthward directed electric field in front of it. This field, potentially, can cause cross-tail current reduction or even disruption.

8. With the tip collapse termed as an "electron trap collapse", the first escaping electrons may be highly energized by the bulk electron population of tip. Certainly under this scenario the energized electrons ought to remain essentially on the closed magnetic field lines.

9. In parallel, during the tip formation and the build up of negative charge, ions are accelerated. It seems that essentially the same electrostatic field acts before and after the tip collapse and accelerates ions and electrons, respectively.

10. Successive pairs of local plasma sheet thinnings/dipolarizations will energize ions and electrons in a repetitive mode.

We assume here that charged particles are distributed along a magnetic field line much like the static electric charges are distributed all over the surface of a conductor. Over a metallic surface, which is considered as an infinite conducting surface, the charge tends to accumulate at sharp points. The consequence is that at such points high surface charge density $p_{s}$ is measured, and the normal electric field strength $E_{n}$ can locally reach extremely high values, $E_{n}=p_{s} / \varepsilon$. The surface charge tries to spread out as much as possible over the surface and a protruding sharp point, by its very nature, is farthest from most of the surface, and so charges are pushed toward it and, once there, are trapped. This phenomenon characterizes the corona and arc discharges, as well as the lightning discharges (Plonus, 1978). Modern technology uses the same principle in satellite ion propulsion systems like the Field Emission Electric Propulsion-FEEP thruster (Maral and Bousquet, 2002), as well as in the "Scanning Tunneling Microscope-STM".

In particular, we consider the similarity between a charged conductor having a protruding tip and a geomagnetic field line having a fully developed sharp point on the midplane and along an already stretched field line. After the tip formation, 
since the ion inertial length is approximately 40 times larger than the electron inertial length (Nagai et al., 2001), ions will easily become unmagnetized at the tip and escape from the magnetic field line. The ion-electron decoupling can produce negatively charged magnetic field lines. The already formed tip further redistributes the negative charge and accumulates a large part of it on the tip. A strong "electrostatic" electric field develops along the magnetic field lines because the electrons basically remain magnetized. In turn, this electric field may play a vital role causing local current disruption (CD) or reconnection; that is, triggering a substorm at large.

With the hydromagnetic theorem of frozen-in magnetic flux the plasma is in motion but has negligible electrical resistance and there are no electric fields in the frame moving with the plasma. Thus, any component of the electric field parallel to the magnetic field must vanish and, in a steady state, the magnetic field lines could be considered equipotentials. Certainly near the sharp tip structure, in a scale length smaller than the ion gyro-radius, the MHD approximation should break down.

Basic energetic particle reviews are the Krimigis and Sarris (1980) and Lopez and Baker (1994). Energetic particle electron-proton counterstreaming activations were found by Sarris et al. (1976), and extensively studied by Sarafopoulos et al. (1984) and Sarafopoulos and Sarris (1987). The concept of repetitive activations throughout a substorm is well established in a work by Sarafopoulos and Sarris (1988): A substorm is composed of many energetic ion bursts, and each of them shows the inverse velocity dispersion feature (IVD), that is the lower energy particles arrive before the higher energy ones. They interpreted the IVD feature as resulting from the finite growth time of the accelerating source. Another important aspect concerning the distribution of energetic particle fluxes within the plasma sheet is the dawn-dusk electron-proton asymmetry which was statistically studied by Sarafopoulos et al. (2001) using the Interball 56-energy channel spectra.

It is generally accepted that the subject of magnetosphere substorms is very complex, many issues remain quite controversial, and the amount of literature is huge. Many substorm models exist, but they may be broadly classified into two categories. One invokes processes in the near-Earth region $\left(X<15 R_{E}\right)$ or some feedback instabilities at the ionospheric altitudes. The other invokes mid-tail magnetic reconnection at $X>15 R_{E}$ to inject plasma earthward, with the braking (i.e. slowing down) of this plasma flow (and magnetic flux pileup under the MHD assumption) as the cause of near-Earth disturbances. The former, is usually referred as the near-Earth current disruption (NECD) model (Lui, 2001), while the latter is usually referred to as the near Earth neutral line (NENL) model that advocates the view of X-line formation to initiate substorm expansion (Baker et al., 1996). It is inevitable that the just mentioned fundamental difference between the two viewpoints would find its way into our discussion.

\section{Observations}

According to the statistical work of Lopez et al. (1989) there are rare events, in the near-Earth $\left(<10 R_{E}\right)$ magnetotail, in which the magnetic field increases at the time of local substorm onset $(\Delta|B|>0)$. Representative examples in this category are the AMPTE satellite events on 28 August 1986, studied by Takahashi et al. (1987), and on 25 April 1985 studied by Lopez et al. (1989). Our extraordinary first event on 4 September 1997 falls in this category, which is characterized by intense north-south turnings of the magnetic field indicating that transient currents in the vicinity of Geotail were strong enough to cancel the Earth's dipole field. Additionally for all the above three mentioned cases $d B_{z} / d t \cong d B / d t$, and the satellite is situated essentially at the neutral sheet, which finally justifies why these events are not detected frequently. Analyzing our first event, we try to get an insight into the particles acceleration mechanism associated with the longstanding question about the role of $d B / d t$ variations. We use data from the Geotail Energetic Particles and Ion Composition (EPIC) instrument (Williams et al., 1994) with time resolution in time series which offer reliable and high-resolution proton and electron measurements simultaneously. Every diagnosed differentiation between the electron and proton behaviour will be our best clue to trace the energization mechanism. The electron head measures fluxes with polar angles $|\theta|<30^{\circ}$, and the two identical proton heads receive fluxes with $9^{\circ}|\theta|<38^{\circ}$. We use the proton differential fluxes from the energetic channels P2 (58$77 \mathrm{keV})$, P3 (77-107 keV), P4 (107-154 keV) and P5 (154$227 \mathrm{keV})$, and the electron integral fluxes for the channel $\mathrm{ED} 1(E \geq 38 \mathrm{keV})$. ED1 provides an eight sector angular distribution every $3 \mathrm{~s}$, while $\mathrm{P} 2$ provides a sixteen sector angular distribution every $6 \mathrm{~s}$.

Three events in the near Earth region are considered absolutely necessary to convey the needed supplementary information concerning the energetic particle acceleration mechanism. Then in order to demonstrate that there are some fundamental similarities between the near Earth activations and the mid- and distant-tail energetic particle responses, we add two more events-substorms that typify the regions at $X=-30.56$ and $-64.2 R_{E}$.

In addition to the EPIC data we extensively use (a) the Geotail magnetic field data obtained by the MGF experiment (Kokubun et al., 1994) having 3-s resolution in the GSM system and (b) the Geotail plasma data generated by taking moments over 12-s intervals of ion distribution functions measured by the low energy particle (LEP) instrument (Mukai et al., 1994). From the GEO Los Alamos National Laboratory (LANL) satellites the 10-s spin averaged energetic particle differential fluxes are used. The LANL satellites spin-axis points toward the center of the Earth. The instrument for the 1990-095 satellite was the Synchronous Orbit Particle Analyzer (Belian et al., 1992). 


\subsection{First event on 4 September (day 247) 1997}

In this case study we are focused on a short interval of four minutes extended from 11:06:40 to 11:10:40 UT of day 247, 1997 (Fig. 1), as it was seen by the Geotail satellite located at $(X, Y, Z)_{\mathrm{GSM}}=(-10.23,-1.38,1.08) R_{E}$, very near the midnight meridian. It is obvious that the peak proton fluxes do not occur simultaneously with those of electrons. We pay attention to the two successive pairs of so called micro-thinning/expansion, or local thinning/expansion geometries of the magnetic field. The two major local thinnings (i.e. transient tail-like structures), which are identified by decreases in the $B_{z}$ and polar angle $\theta$ traces of the magnetic field (third and fourth panels, respectively), are centered around 11:08:05 and 11:09:20 UT. Each stretched topology of the magnetic field is followed by a local plasma sheet expansion corresponding to a local dipolarization; terms used for instance by Lopez et al. (1989). These fluctuations are intense and the overall excursion $\Delta B_{z}$ is twice as much the initial $B_{z}$ magnitude. We emphasize the fact that the ratio $B_{z} / B_{\text {total }}$ is close to 1 (bottom panel) and, therefore, as it is already stressed the event is extraordinary. Before the fluctuations the satellite was situated near the neutral sheet $\left(B_{x} \cong-5 \mathrm{nT}\right)$. The interval under study is part of an isolated substorm, as it is evident from the sharp energetic particle injection seen by LANL 1990-095 and the AE index (not shown here). Moreover, this substorm event is included in other research studies (Lui, 2001).

Most importantly, the magnetic field fluctuations are tightly associated with energetic particle fluxes. The energetic proton differential fluxes (first panel, P2 channel: 58$77 \mathrm{keV}$ ) essentially increase at local thinnings. In contrast, the energetic electron integral fluxes (second panel, ED1 channel: $E>38 \mathrm{keV}$ ) mainly increase at local expansions. Therefore, Geotail detects a local injection of energetic particles with the electron population being delayed by $\sim 40 \mathrm{~s}$. However, this delay is small in order to destroy the "dispersionless" character of the injection: We mention that, for instance, Friedel et al. (1996), have classified as dispersionless injections those having less than 4 min dispersions, while Lopez et al. (1990) have compiled a catalog of 167 dispersionless injections where the flux increase in any two of the channels was displaced by no more than 72-s.

We evaluate that the above detected different occurrence times between peak proton and peak electron fluxes is a major finding related to the fundamental magnetospheric dynamics. The non-simultaneous production of protons and electrons is further emphasized in Fig. 1 using the abbreviation HF (high fluxes) for the peak fluxes. Our interpretation scheme is given latter on. It should be underlined that the Fig. 1 measurements are with the highest available resolution, which readily makes clear why the $58-77 \mathrm{keV}$ energetic proton fluxes apparently show a periodicity of $6 \mathrm{~s}$. The latter is due to the satellite spin period of $3 \mathrm{~s}$ : A complete angular distribution on the ecliptic plane is sampled every $3 \mathrm{~s}$; although one out of two angular distributions is transmitted through the telemetry subsystem. Therefore, the 3-s or 6-s periodicity in flux profile time series is an excellent indicator for the anisotropy of energetic particles; the presented proton profile shows a profound streaming character. Certainly, it must be underlined that we always pay attention to the envelope of higher flux values. The ED1 channel of energetic electron fluxes provides an 8 sector angular distribution every $3 \mathrm{~s}$.

At 11:07:50 UT the plasma sheet locally begins to thin, the near-Earth plasma sheet probably narrows in the north-south direction, the magnetic field becomes more tail-like and the energetic electron fluxes show a clear reduction. It seems that Geotail finds itself on higher latitude field lines that have less energetic electron content, although at the same time, the energetic proton fluxes increase by more than one order of magnitude. The electron flux reduction is probably due to the magnetic field reconfiguration; in sharp contrast, the enhanced proton fluxes are probably freshly accelerated populations. At thinnings although the magnetic field decreases the proton fluxes increase, which provides evidence for a nonadiabatic process at work.

The energetic electrons are accelerated during the dipolarizations occurred around the times 11:08:40 and 11:09:50 UT. It is worth noticing that the freshly accelerated electrons show double-peaked angular distributions (not shown here) and probably stay on closed magnetic field lines characterized by latitude angles $\theta \geq 70^{\circ}$.

The energetic proton fluxes for the first peak (11:07:45 to $11: 08: 25$ UT) of Fig. 1 are clearly earthward, whereas later, and especially for the third peak, they are changed to tailward. However, we have to take into account and subtract the contribution to the proton anisotropy which is due to the plasma convection velocity. We apply ComptonGetting transformations to achieve angular distributions in the plasma frame of reference. The Compton-Getting transformation of the proton intensities to the plasma frame of reference moving with a velocity $\mathrm{V}$ is given by Gold et al. (1975), while the same methodology is applied in the work of Sarafopoulos and Sarris (1987). The ambient convection velocities $V$ are taken $\sim 700$ (earthward) and -100 (tailward) $\mathrm{km} \mathrm{s}^{-1}$ for the first and third peak fluxes of Fig. 1, respectively. The $V_{x}$ component of plasma velocity is displayed in the fifth panel trace of Fig. 1. For the plasma frame of reference, presumably, the only resulting anisotropy would be due to the effect of the accelerating electric field. We compute the spectral index $\gamma$ for the channels P2 with P3, P4 and P5. It is assumed for each pair of differential intensities a power law, which is expressed by

$j_{2} / j_{n}=\left(E_{2} / E_{n}\right)^{-\gamma}$.

This way the $\gamma$ is computed to be $\sim 7.6$ for the $(\mathrm{P} 2, \mathrm{P} 3)$ pair, 5.44 for the $(\mathrm{P} 2, \mathrm{P} 4)$ pair and 5.6; actually we do not need the energy spectrum to be strictly a power law. Two representative angular distributions, over the ecliptic plane XY, from 


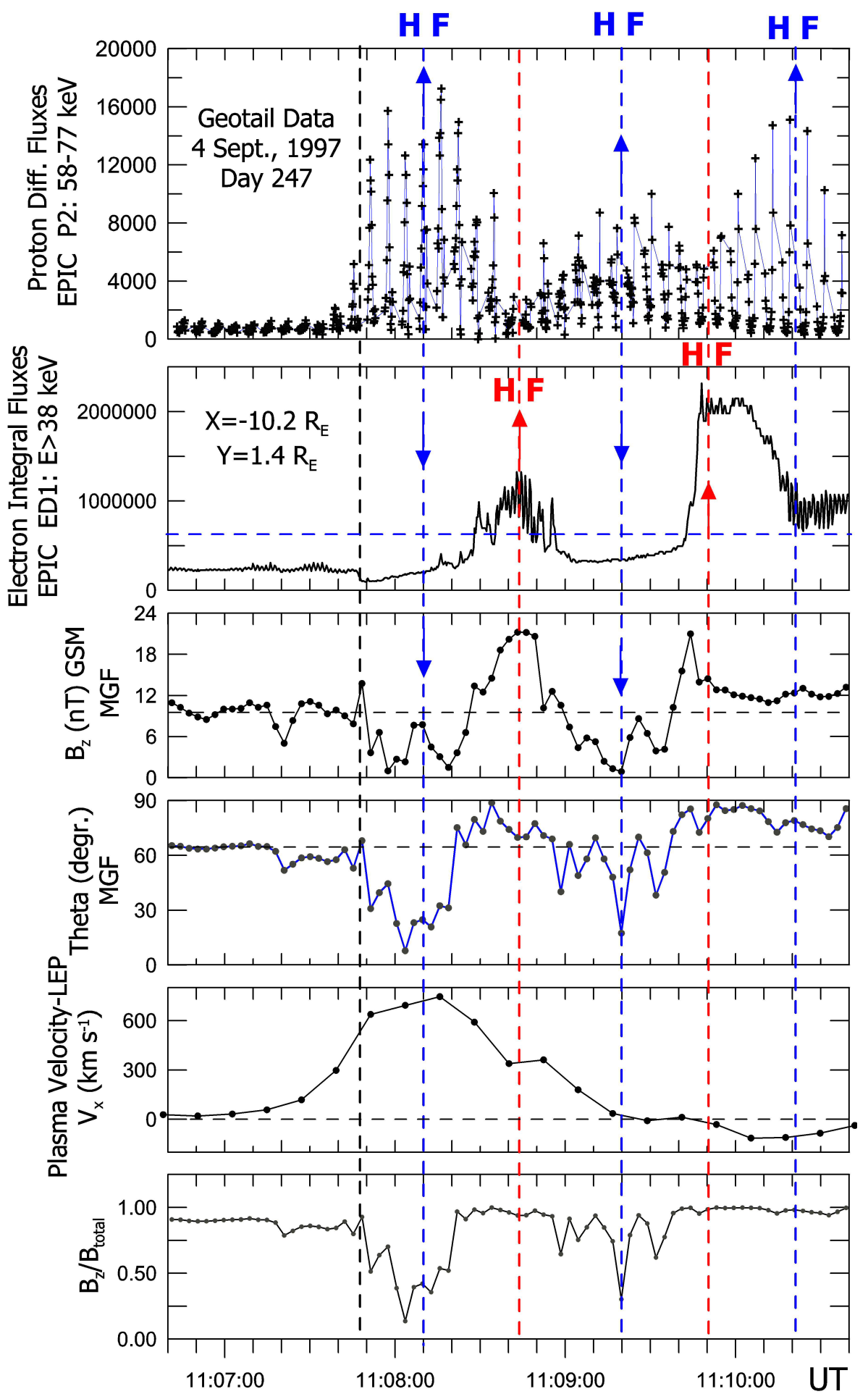

Fig. 1. A 4-min interval showing Geotail data sets of day 247, 1997. From top to bottom, proton differential fluxes (58-77 keV), electron integral fluxes $(E \geq 38 \mathrm{keV}), B_{z}$ (in nTs) and polar angle $\theta$ (in degrees) of the magnetic field, $V_{x}$ component of plasma velocity (km s ${ }^{-1}$ ), and the ratio $B_{z} / B_{\text {total }}$. The abbreviations HF mean high fluxes. The blue and red vertical dashed lines mark the peak proton and electron fluxes, respectively; the black vertical dashed line marks the start time for the first local plasma sheet thinning. 


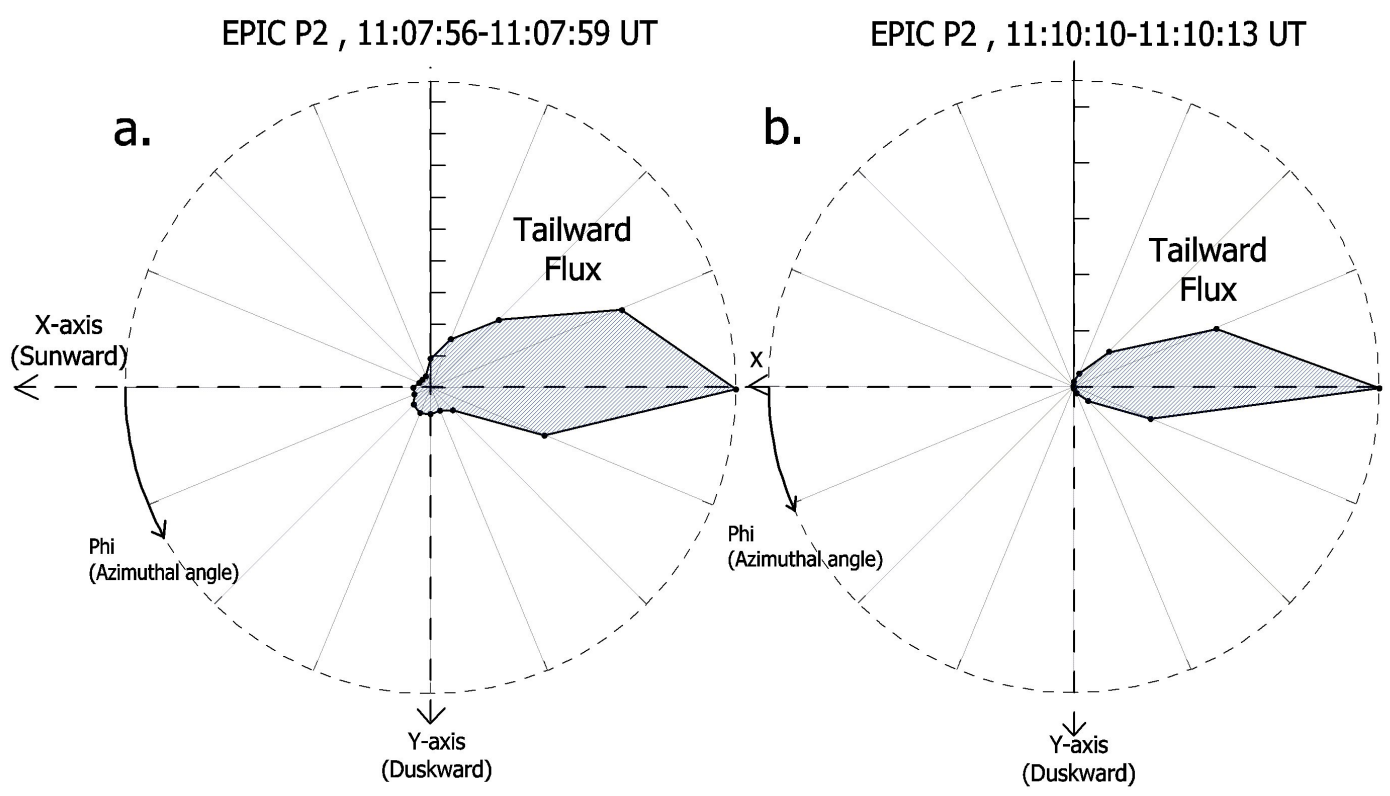

Fig. 2. Typical angular distributions for the $58-77 \mathrm{keV}$ energetic proton fluxes of Fig. 1 in the plasma frame of reference over the XY plane. The (a) and (b) angular distributions are representative of those observed in the first and third peak fluxes of Fig. 1. The sampled flow direction or otherwise the azimuthal angle phi (in degrees) points toward Sun (tail) with $\phi=0^{\circ}\left(180^{\circ}\right)$. The satellite rpm is 20 .

those transformed with $\gamma=5.5$ and corresponding to the first and third peak fluxes of Fig. 1, respectively, are shown in Fig. 2. The azimuthal phi angle shows the proton flow direction that equals zero $\left(180^{\circ}\right)$ for earthward (tailward) fluxes. It is worth noticing that the earthward fluxes in the "laboratory" frame of reference are really tailward in the plasma frame of reference, whereas the initially tailward fluxes are slightly affected. If we input the somewhat lower plasma velocity $V=600 \mathrm{~km} \mathrm{~s}^{-1}$ or a lower spectral index $\gamma=4.5$ an almost similar flow reversal will be attained. Certainly a higher index $\gamma$ further increases the anisotropy for the transformed distribution. Therefore, we infer that the energetic proton fluxes are accelerated earthward of the Geotail site.

To summarize our main points:

1. Burst-like energetic electron and proton fluxes are not produced simultaneously. A complete elementary increase-then-decrease in proton or electron fluxes lasts $\sim 1$ min.

2. Freshly accelerated energetic proton fluxes (streaming tailward) are produced at local thinnings $\left(B_{z} \cong 0 \mathrm{nT}\right)$.

3. Freshly accelerated energetic electron fluxes are produced at local dipolarizations $\left(B_{z}=12-21 \mathrm{nT}\right)$ and remained trapped on closed magnetic field topologies.

4. A prolonged thinning of the plasma sheet with $B \cong B_{z} \cong 10 \mathrm{nT}$ at $X=-10.2 R_{E}$ is preceded the onset of energetic protons. The detected $\Delta B_{z} \cong B$ fluctuations are associated with extremely sharp magnetic field reconfigurations.

\subsection{Second event on 4 September (day 247) 1997}

This near Earth event occurs at $(X, Y, Z)_{\mathrm{GSM}}=(-10,-0.2$, $0.5) R_{E}$ and precedes the already analyzed period. Thus, in this situation the observed energetic particle accelerations belong either to a substorm pseudo-breakup or to a very weak substorm, given that for this interval the $\mathrm{AE}$ index and the energetic particle fluxes from the LANL geostatic satellites alike (not shown here) remain unvaried. We have plotted an interval of $5 \mathrm{~min}$, in Fig. 3, with the same format as in Fig. 1. In particular we pay attention to the $\sim 1$ min interval from $\sim 10: 23$ to $\sim 10: 24 \mathrm{UT}$, since this period is associated with a satellite passage from the plasma regime characterized by distinct negative excursions in $B_{Z}$ and $\theta$ to a more dipole-like magnetic field structure. The most prominent feature is that the highest proton fluxes (marked as HF, top panel) precede those of the highest electron fluxes (marked as HF, second panel). The proton short-lived peak flux shows an increase of about one order of magnitude. The major increase in $\theta$ occurred at $\sim 10: 24 \mathrm{UT}$, while at that time the $B_{Z}$ tends to become equal to the total $B$.

From 10:20:00 to 10:21:20 UT the energetic protons clearly show earthward fluxes probably since they are strongly affected by the high plasma velocity $V_{x}(420-$ $700 \mathrm{~km} \mathrm{~s}^{-1}$, fifth panel) much like as in the preceded event. From 10:22:30 to 10:24:00 UT, while the $V_{x}$ smoothly decreases from 0 to $-300 \mathrm{~km} \mathrm{~s}^{-1}$, the protons undoubtedly stream tailward. At this point we believe that it is not insignificant to make an additional comment: From 10:22:40 to 10:23:15 UT, although we observe the highest proton fluxes, 
weak electron fluxes are also detected streaming tailward. The latter is a very significant observation which is also identified in the next case study and presented using proton angular distributions.

\subsection{Third event on 9 March (day 68) 1995}

\subsubsection{First subinterval}

During this $\sim 15 \mathrm{~min}$ interval Geotail was positioned at $(X, Y, Z)_{\mathrm{GSM}}=(-12,-6,-1.7) R_{E}$. Data sets are shown in Fig. 4 with almost the same format as in the preceded cases, and we stress the following prominent points:

1. A burst of energetic proton fluxes (marked as HF, top panel) occurs at 05:41:30 UT, whereas a simultaneous energetic electron burst is not detected. The proton fluxes are multiplied by $\sim 20$, and at this particular time, the electrons do not respond. The peak proton fluxes occur with the major negative $B_{Z}$ excursion.

2. From $\sim 05: 40$ to $05: 46$ UT the magnetic field inside the plasma sheet is highly disturbed and the same does the local plasma velocity (bottom panel) showing abrupt variations. In this figure, instead of plotting the parameter $B_{z} / B_{\text {total }}$, we have preferred to include the $B_{X}$ trace. One reason is that the $B_{X}$ shows that the satellite is repetitively crossing the neutral sheet, while an additional reason is referred below. Decreases in $B_{Z}$ are frequently observed, although at the same time the average $B_{Z}$ and the electron fluxes enhance. Finally, without any doubt the highest electron fluxes are not associated with a similar peak in proton fluxes. Certainly we have to note that in this event we fail to precisely determine the time when the satellite leaves the one plasma regime, which is characterized by $-B_{Z}$ excursions, and enters into another one with more dipolelike geometry. As a matter of fact, this crossing seems to be a long-lasting one. Nevertheless, the dipolarization process is under development and this is better demonstrated around 05:44 UT, when although we observe the same average value of $\theta$, being $\sim 40^{\circ}$, however, the $B_{X}$ component is larger; the satellite goes away from the neutral sheet and, therefore, the dipolarization process is in progress.

3. It is remarkable that the major peak proton fluxes at $\sim 05: 41: 30$ UT are not associated with similar peak electron fluxes. This time the energetic electron population remains in a low level and flows tailward along the magnetic field lines. The electron fluxes, over the ecliptic plane XY, are modulated by the satellite revolution period; and thus we know the electron flow direction every $3 \mathrm{~s}$. Data from almost six satellite revolutions are plotted in Fig. 5 (top panel) showing that the four major peak fluxes (marked with arrows) occur antisunward.

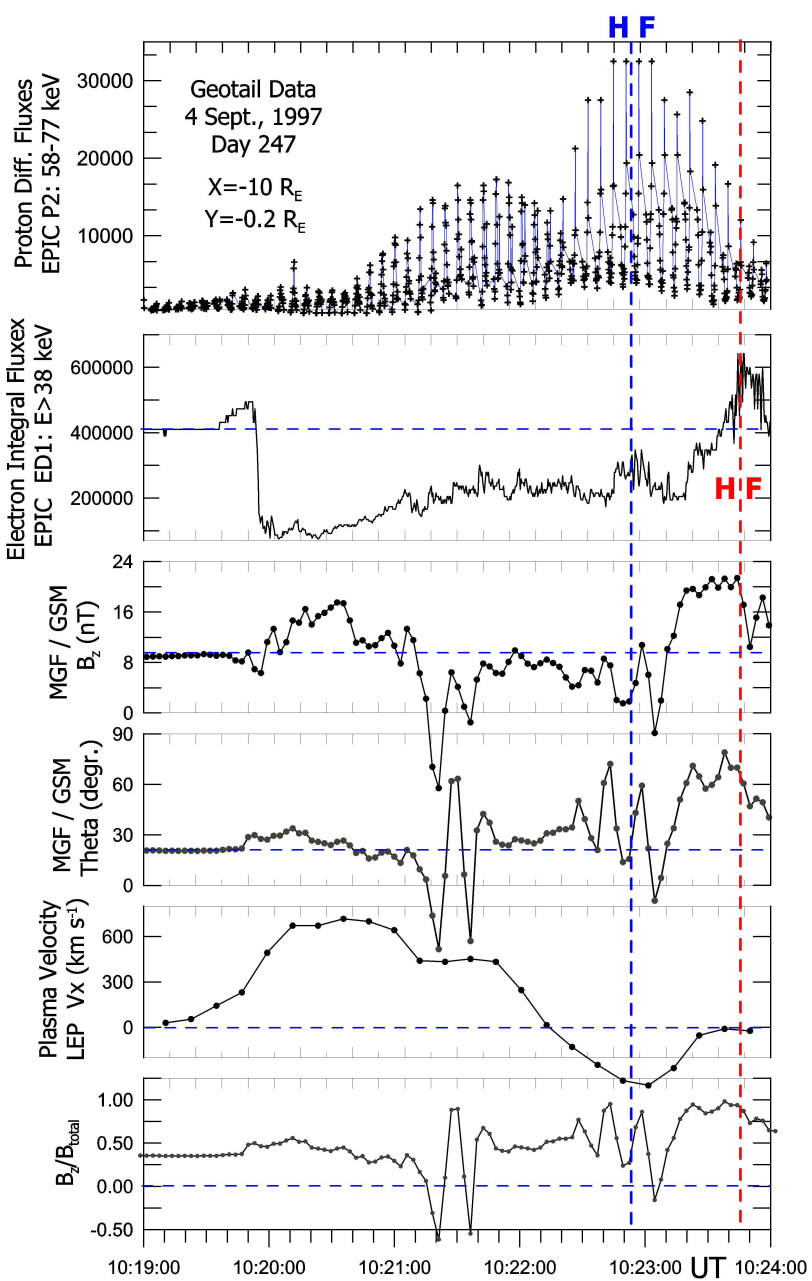

Fig. 3. A 5-min interval of day 247, 1997. Same format as in Fig. 1. The blue and red vertical dashed lines mark the highest proton and electron fluxes, respectively.

The azimuthal angle phi (second panel) is the same used in Fig. 2.

4. In parallel to electrons, energetic proton fluxes also stream tailward (i.e. maximum fluxes occur with $\phi=180^{\circ}$, third and fourth panels), and along the magnetic field lines. Proton fluxes for six consecutive satellite revolutions are shown in Fig. 5. The plasma velocity $V_{x}$ is very low and does not affect the proton angular distributions. Finally an explanation is demanded coupling the simultaneously observed high tailward proton fluxes and low tailward electron fluxes. Latter on, in the discussion section, we will exhibit our interpretation.

5. The first major dipolarization seen along the $\theta$ trace (fourth panel of Fig. 4) at $~ 05: 34$ UT is accompanied by increased electron fluxes (second panel). Moreover, we observe four successive dipolarizations, which are marked with the letters $\mathrm{a}, \mathrm{b}, \mathrm{c}$ and $\mathrm{d}$, corresponding to 


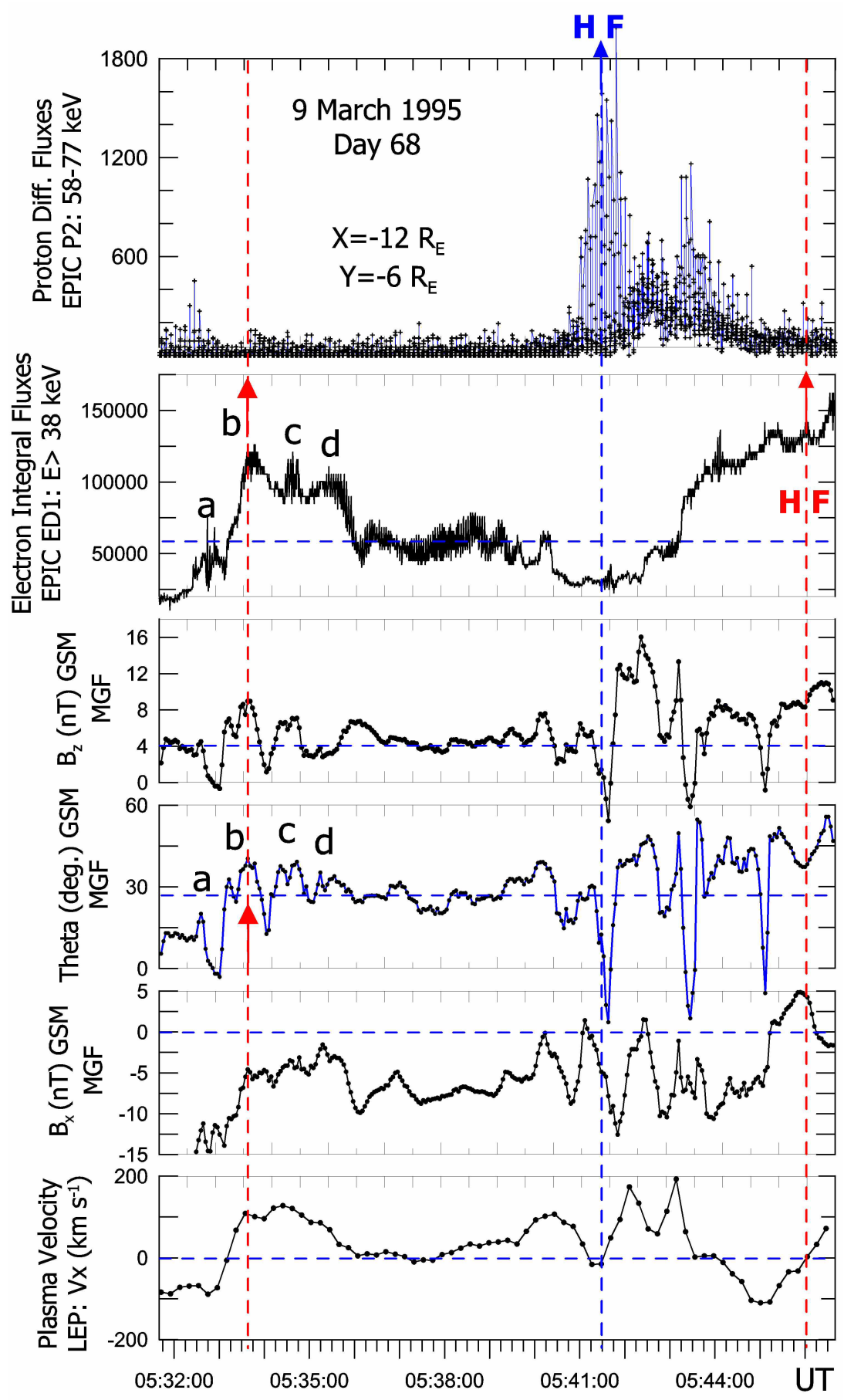

Fig. 4. A 15-min interval of day 68, 1995. Almost same format as in Fig. 1. The blue and red vertical dashed lines mark the highest proton and electron fluxes, respectively.

distinct electron flux increases; the proton fluxes do not respond and, therefore, energetic protons and electrons are not produced simultaneously.

\subsubsection{Second subinterval}

The just studied "first subinterval" of day 68, 1995, with its intense local energizations, belongs to a distinct and isolated substorm having at least one more worth studying subinterval. This substorm follows a period of at least twelve hours 


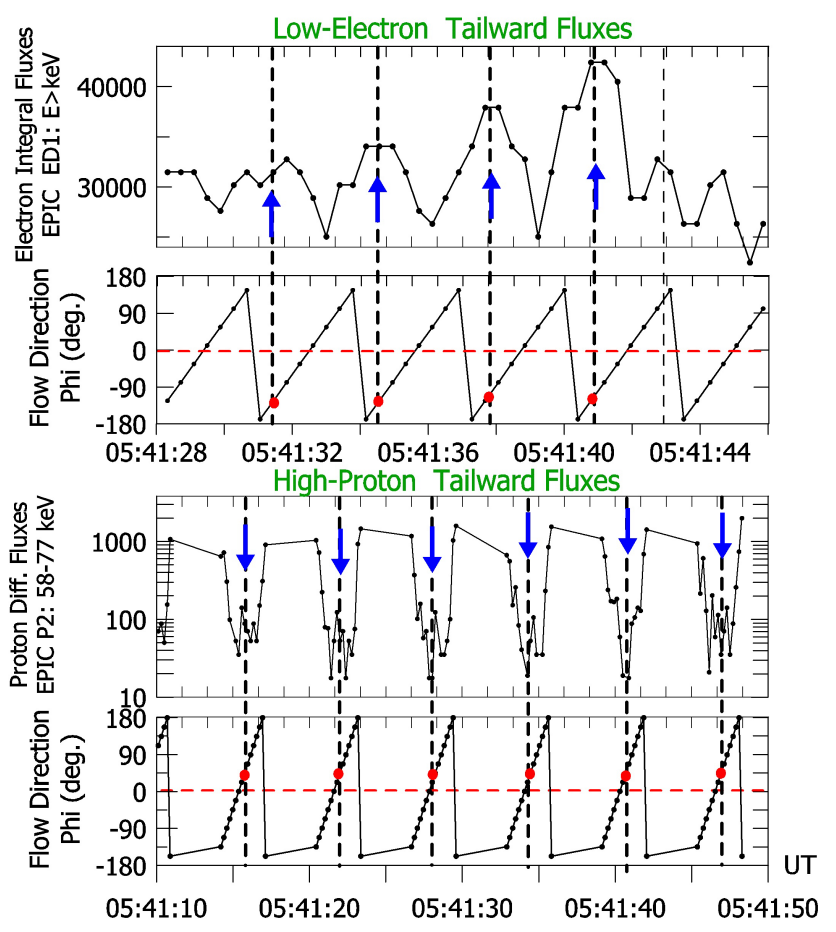

Fig. 5. Energetic electron (top two panels) and proton fluxes from the Geotail/EPIC instrument streaming tailward. The flow direction phi (in degrees) points toward Sun (tail) with $\phi=0^{\circ}\left(180^{\circ}\right)$ and is the same angle as it is used in Fig. 2. The vertical dashed lines emphasize the tailward character of flow.

without any detectable energetic electron injection seen by the four geostationary satellites 1984-124, 1991-080, 1989046, and 1990-095. Additionally, for the same period the $\mathrm{AE}$ index remains unvaried and extremely low, typically less than $40 \mathrm{nT}$ (not shown here). Highlighting the possible particle acceleration mechanism, we focus on the subinterval of Fig. 6 characterized by a Geotail smooth crossing of the whole plasma sheet, from the north to the south lobe. The satellite encounters the neutral sheet at 04:56:30 UT (thickdashed vertical line), when the $B_{x}$ component changes sign (third panel), the polar angle of the magnetic field reaches its maximum value ( $\theta \cong 28^{\circ}$, fourth panel), and the field strength affected by the plasma diamagnetic effect becomes almost zero (fifth panel). During this plasma sheet crossing, the geostationary satellite LANL 1990-095, which is located $38.75^{\circ}$ eastward of local midnight, identifies the local growth phase of a substorm. The latter is inferred from the 50$75 \mathrm{keV}$ energetic electron fluxes which steadily diminish until 05:06 UT (bottom panel), when the local substorm onset occurs producing an energetic electron injection. The Geotail passage through the plasma sheet reveals a symmetric magnetic field structure corresponding to a systematic increasethen-decrease of energetic electron fluxes (second panel), which are maximized at the neutral sheet. Most importantly, under these circumstances none could anticipate, during this

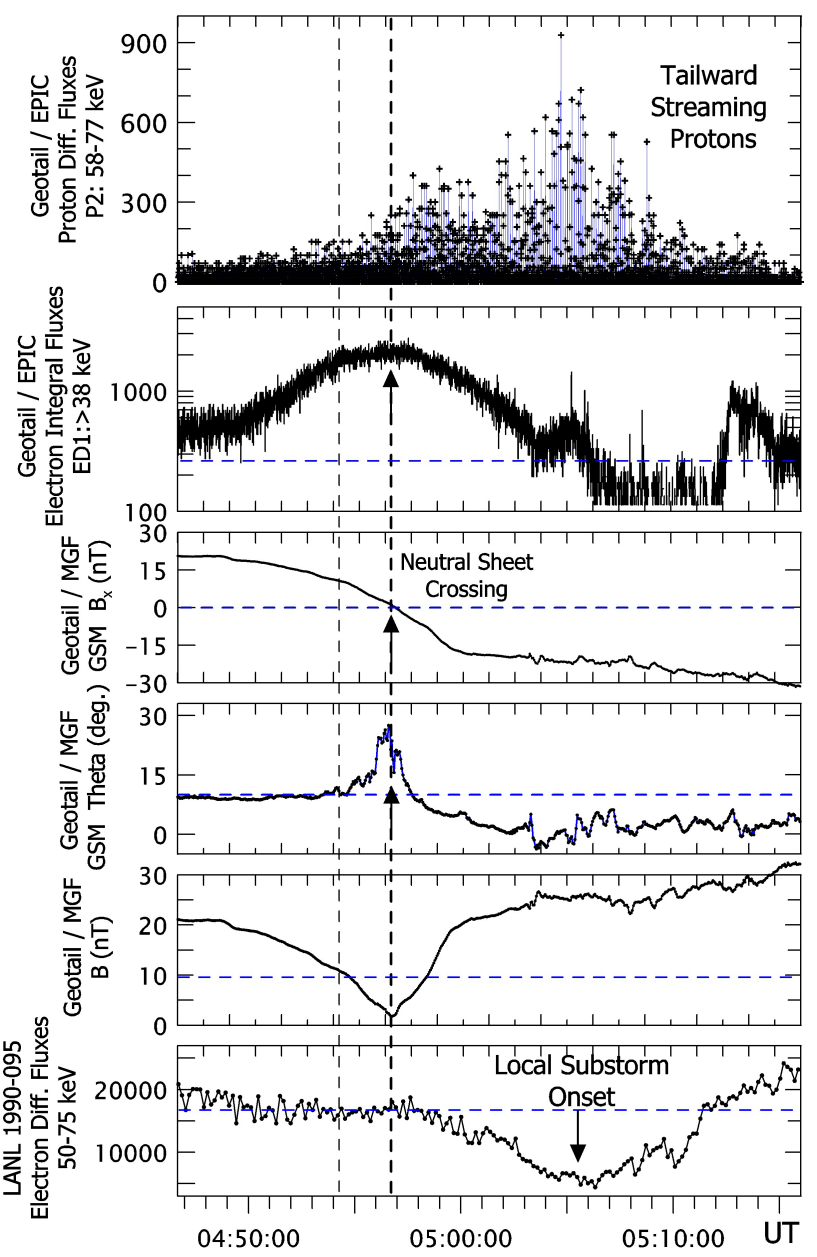

Fig. 6. A smooth plasma sheet crossing. Geotail data sets from top to the fifth panel: Proton differential fluxes $(58-77 \mathrm{keV})$, electron integral fluxes ( $E \geq 38 \mathrm{keV}$ ), $B_{x}$ (in nTs), polar angle $\theta$ (in degrees) and strength $\mathrm{B}$ of the magnetic field. The bottom panel shows 50$75 \mathrm{keV}$ energetic electron differential fluxes from the LANL 1990095 satellite.

crossing, an active particle energization mechanism at work; all the variations seem to have a spatial character. However, just before the neutral sheet encounter, at the moment marked with a thin-dashed vertical line, we clearly discern counter-streaming field-aligned energetic electron and proton populations which, presumably, are due to an in situ accelerating process. Figure 7 shows the ED1 channel integral energetic electron fluxes (top panel) associated with 10 consecutive spacecraft revolutions (second panel). The electron fluxes for $\sim 30 \mathrm{~s}$ are systematically modulated since they stream sunward $\left(\phi \cong 0^{\circ}\right)$. The ten repetitive cycles of sunward streaming electrons provide strong evidence for a short-lived active acceleration mechanism that monotonically increases the electron fluxes (see the polynomial fitting, blue-dashed line in top panel). For the same period, the P2 channel of energetic proton differential fluxes (third panel) shows tailward 

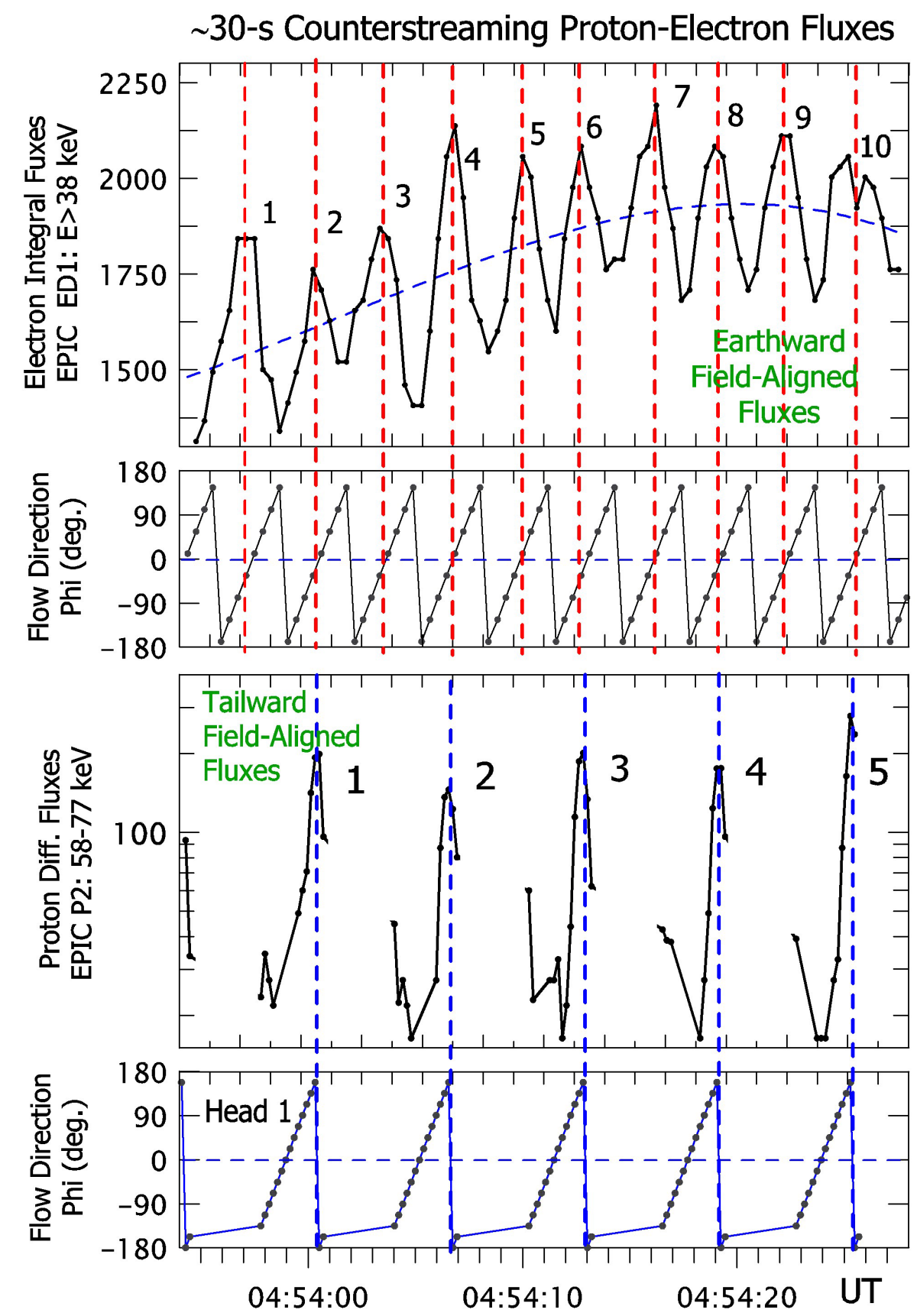

Fig. 7. An interval with $\sim 30$-s (i.e. ten satellite revolutions) counterstreaming field aligned fluxes of energetic protons and electrons obtained by the Geotail/EPIC instrument; same format as in Fig. 5 . Proton and electron fluxes are slightly smoothed by a running window of $\sim 1 \mathrm{~s}$. The red and the blue vertical dashed lines mark the peak electron and proton fluxes, respectively.

fluxes ( $\phi \cong 180^{\circ}$, bottom panel). The available consecutive angular distributions are numbered along the flux traces for both electrons and protons. Both proton and electron fluxes in Fig. 7 are slightly smoothed by a running window of $\sim 1 \mathrm{~s}$.

Additionally, for this substorm, Wanliss et al. (2000) determined via ground magnetograms and meridian scanning photometer data that the expansion phase onset occurred at
04:59 UT. On the basis of their timing the particle acceleration presented in Fig. 7 occurs $\sim 5$ min before the substorm onset. Therefore, at that time, before any current disruption or reconnection onset, the only mechanism producing energetic particles is most probably the "tip structure". Moreover, tailward streaming energetic protons are clearly measured as early as at 04:50 UT: it is difficult to imagine an inductive 


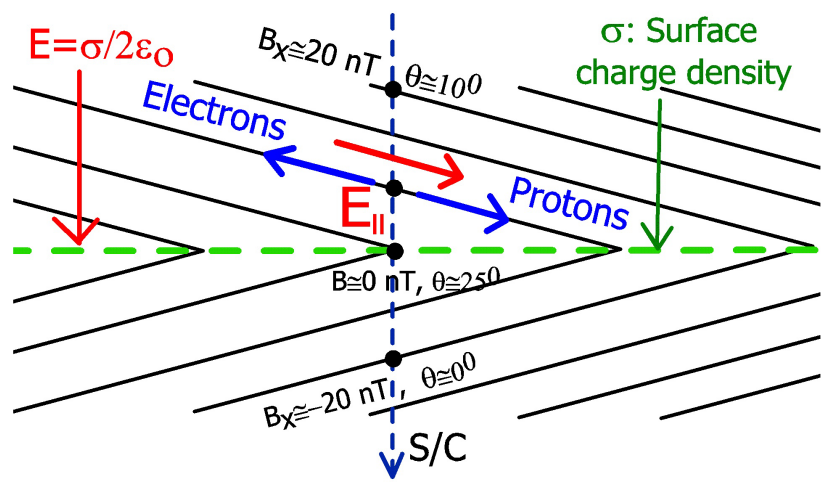

Fig. 8. Schematic illustrating a locally thinned plasma sheet; negative charge is accumulated in the tip structure, and the surface charge density $\sigma$ eventually produces the electric field along the magnetic field lines. The latter is considered responsible for the observed counterstreaming energetic proton and electron fluxes shown in Fig. 7.

electric field accelerating particles 9 min before the substorm expansion phase onset.

We now describe an interpretation scheme for the bidirectional electron-proton fluxes shown in Fig. 7. We assume that a tailward-pointing, field-aligned electric field $E_{\|}$, which accelerates these particles, develops earthward of the satellite on the sampled magnetic field lines. Figure 8 schematically illustrates the local particle acceleration mechanism which is based on a negatively charged neutral sheet region. The latter is probably due to the charge accumulation at the tip of each stretched magnetic field line in a TCS. In this perspective, above and below the neutral sheet, an electric field of magnitude $E=\sigma / 2 \varepsilon_{o}$ arises due to "an infinite sheet of uniform charge" with negative surface density $\sigma$ over the tail midplane. The component $\mathrm{E}_{\|}$being parallel to the magnetic field lines will accelerate electrons and protons in opposite directions. With a polar angle of the magnetic field $10^{\circ}-20^{\circ}$, a thinned plasma sheet having length $1-2 R_{E}$ and a total electric field $100 \mathrm{mV} / \mathrm{m}$, the estimated $E_{\|}$will be $17-34 \mathrm{mV} / \mathrm{m}$ and the overall energization will be 110 $440 \mathrm{keV}$. We have hypothesized that the energetic protons become unmagnetized in the thinned plasma sheet and move tailward, something that is actually observed throughout the Fig. 6 interval. The protons move tailward and undergo energization by the accelerating electric field in the TCS region.

However, the mechanism is not fully developed here, at least for the electrons, given that much higher electron fluxes are produced later with the occurring local plasma sheet dipolarizations. Progressively, as the substorm evolves the polar angle of the magnetic field increases from the initial level of $10^{\circ}$ at $\sim 04: 50 \mathrm{UT}$, to $\sim 30^{\circ}$ at $05: 35$, and $\sim 45^{\circ}$ at $\sim 05: 45 \mathrm{UT}$; the energetic electron fluxes increase 30 and 50 times, respectively. Conversely, the energetic proton fluxes increase no more than $\sim 3$ times as the substorm grows.

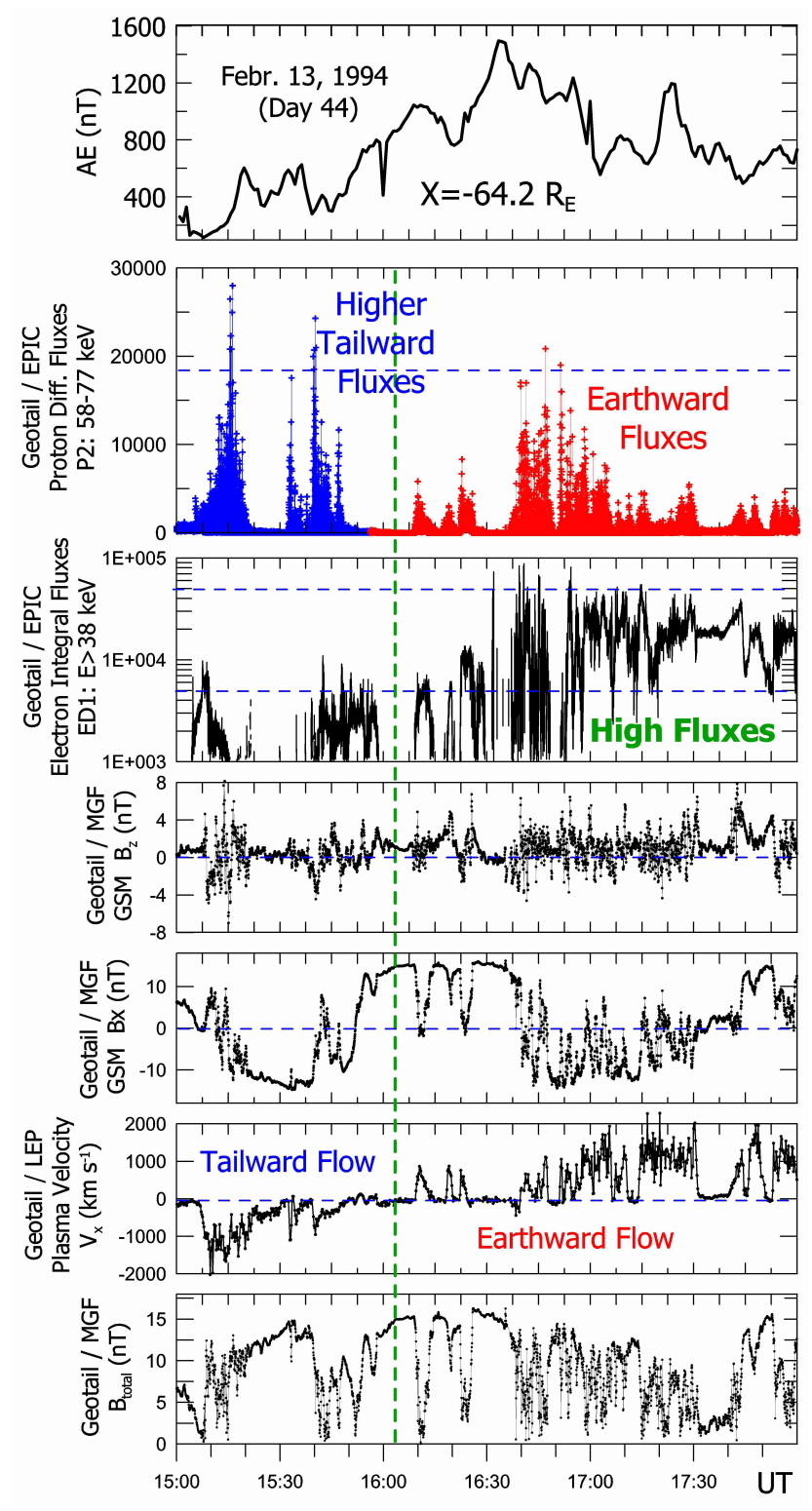

Fig. 9. An isolated substorm as has been observed on 13 February (day 44), 1994, by Geotail at $X=-64.2 R_{E}$. Same format as in Fig. 1, except that the geomagnetic activity index AE is added in the top panel trace. The tailward energetic proton fluxes (second panel, blue line) are distinguished by the earthward ones (red line). The green-dashed vertical line marks the time on which the plasma changed its flow direction.

Therefore, the observed proton populations at $\sim 05: 00 \mathrm{UT}$ are not an insignificant fraction of those measured after the substorm expansion phase. It seems that the main energization mechanism of protons is triggered before the energization mechanism of electrons; further discussion will take place later on in the appropriate subsections. 
2.4 Fourth event on 13 February (day 44) 1994: a distant tail case study

There are some fundamental similarities between the near Earth observations and those in the distant tail. This case study typifies the distant Earth situation, and we suggest that the same underlying energization mechanism is at work near and far from Earth. We present a typical substorm observed at $(X, Y, Z)_{\mathrm{GSM}}=(-64.2,8.7,-1.4) R_{E}$ that shows a remarkable differentiation in the proton-electron response. Although a 3-h interval is exhibited here (Fig. 9), we believe that the same information is carried as in Fig. 1 treating a period of 4-min. In the distant tail, the plasma sheet recovery occurs with the substorm recovery phase, while the acceleration source retreats tailward; the substorm associated dipolarization is in progress. We underline the main points for the under study substorm as follows:

1. The AE index (top panel of Fig. 9), synthesized from ground station magnetograms, documents the time history of this intense (maximum value of $1500 \mathrm{nT}$ ) substorm.

2. Initially the near Earth acceleration source produces tailward proton fluxes at Geotail (second panel, blue line), whereas the proton fluxes are switched to earthward (second panel, red line) as the source retreats tailward.

3. The plasma flow velocity (sixth panel), with extremely high values ( $V_{x}$ often being greater than $1000 \mathrm{~km} \mathrm{~s}^{-1}$ ), changes its flow direction as the energetic proton fluxes do.

4. When the plasma flow changes its direction, the satellite spends most of its travel time in the north lobe domain because the plasma sheet is probably thinned and the retreating tip structure encounters the satellite.

5. The acceleration mechanism seems to be slightly more effective in energizing tailward than earthward proton fluxes. The tailward to earthward flux ratio is less than 2.

6. The situation is radically different for the energetic electron fluxes: As the source was positioned earthward of spacecraft the electron fluxes were low, whereas with the source tailward of spacecraft 10 times greater electron fluxes were counted.

7. Electrons essentially remain magnetized and are accelerated on closed field lines associated with the plasma sheet recovery. In contrast, protons can easily escape from the closed geometry and diffuse in the nearby magnetic field lines.

8. At times, during the plasma sheet recovery phase, we observe energetic protons and electrons streaming earthward simultaneously. Such a period is from
16:47:50 to $16: 48: 10 \mathrm{UT}$ and an explanation is given bellow.

The similarity with the near Earth observations is that in front of the accelerating source (i.e. the satellite is located tailward of the source) mainly energetic protons are produced, whereas behind it energetic electrons are measured.

\subsection{Fifth event on 29 January (day 29) 1998: a mid-tail case study}

In this example (Fig. 10) the satellite remained near the current sheet throughout the substorm, a rare occurrence. Geotail being positioned at $(X, Y, Z)_{\mathrm{GSM}}=(-30.56,0.55$, $-2.53) R_{E}$ clearly reveals the plasma sheet thinning and recovery phases associated with an isolated moderate substorm. The latter is evident from the BJN (Bear Island) ground magnetogram of the IMAGE array shown in the top panel trace of Fig. 10. The BJN station with geographic latitude and longitude $74.5^{\circ}$ and $19.2^{\circ}$, respectively, is located close to the midnight meridian and clearly shows a sharp magnetic field reduction caused by intense electrojet currents. The thinning phase is characterized by intense and persistent tailward plasma flows (sixth panel) and recurring negative turnings along the $B_{z}$ component trace (fourth panel). In contrast during the recovery phase the flow is reversed and the $B_{z}$ is dominated by multiple positive turnings. The ongoing thinning phase gradually changes the magnetosphere configuration and the satellite exits into the south lobe structure, from which it returns to the central plasma sheet during the recovery phase (fifth panel). An insert of the AE index from 21:00 to 24:00 UT is shown emphasizing the recovery phase of this substorm. Most importantly, the thinning phase is dominated by intense tailward-blue coloured proton fluxes, and the recovery phase is dominated by high electron fluxes. The reader should note that the time series are in logarithmic scales; hence the ratio of tailward to earthward proton fluxes is $\sim 10$. Therefore, we infer that protons and electrons are energized essentially separately. At different times, although the satellite essentially remains in the same central plasma sheet region, we observe either overwhelming energetic proton populations (thinning phase), or extremely high energetic electron populations (recovery phase). Presumably, the just expressed implication largely supports our main scenario in this work. The tailward retreating tip structure encounters the satellite at $\sim 23: 00 \mathrm{UT}$, when the plasma sheet is profoundly thinned. 


\section{Discussion}

\subsection{Electron and ion heating mechanism}

\subsubsection{The demand for a new acceleration scheme}

In general, particle acceleration is produced by electric fields or by time-varying magnetic fields; the rate of energy gain is given by

$\frac{d W}{d t}=q \bar{v} \cdot\left(\bar{E}_{\text {ind }}+\bar{E}_{e l}\right)+\mu \frac{\partial B}{\partial t}$

where the term $\mu \partial B / \partial t$ is referred to the betatron acceleration being significant when the time scale of magnetic field variation $\left(T_{m f}\right)$ is much greater than the particle gyroperiod $\left(T_{\mathrm{gyr}}\right)$. That is, $T_{m f} \gg T_{\mathrm{gyr}}$, while the first adiabatic invariant, $\mu$, is conserved. Strong inductive electric fields $\bar{E}_{\text {ind }}$ parallel to the particle velocity $\bar{v}$ may accelerate particles when the $T_{m f}$ is comparable to $T_{\mathrm{gyr}}$. Additionally, electrostatic electric fields may contribute to the rate of energy gain via the term $q \bar{v} \cdot \bar{E}_{e l}$. Certainly, in the studied cases, inductive or electrostatic electric fields may be at work since the energetic protons become non-adiabatic in a stretched magnetic field topology. However, all these mechanisms will produce protons and electrons simultaneously, something which is not observationally supported. The latter is a major criterion leading us to introduce the "asynchronous mode" proton-electron acceleration mechanism. We systematically observe different time response for protons and electrons: Near Earth proton peak fluxes correspond to local $B_{z}$ minima (thinnings), whereas electron peak fluxes occur with local $B_{z}$ maxima (dipolarizations). More distant from Earth, the substorm thinning phase is dominated by high proton and weak electron fluxes, whereas the substorm recovery phase is characterized by vast energetic electron fluxes. Therefore, we infer that the energization mechanism seems not to be controlled, in a large degree, by the factor $d B / d t$, which is mainly associated with the magnetic field reconfiguration. Conversely, the operating mechanism preferentially accelerates protons at local thinnings and electrons at local dipolarizations.

We frequently observe $d B / d t$ decreases occurring immediately after $d B / d t$ increases. In this situation, if we suppose according to Faraday's law an accelerating electric field, then it and the energetic protons flow alike should reverse their directions. The latter is never observed; for instance in Fig. 1 the $d B / d t$ factor irrespective of its sign (i.e. negative at 11:07:50 and positive at 11:08:40 UT) produces proton fluxes directed earthward.

We have simultaneously observed energized proton and electron fluxes without any $d B / d t$ variation: on day 68,1995 , the satellite goes through a stationary TCS structure; however, oppositely streaming field-aligned energetic proton and electron populations are observed. Energetic protons streaming tailward are clearly observed for $9 \mathrm{~min}$ before the substorm onset. This provides strong evidence for an existing active acceleration mechanism which gives rise to an electric field $E_{\|}$parallel to the magnetic field lines.

To summarize, energetic proton and electron populations are not primarily produced by $d B / d t$ variations, since

(a) proton and electron fluxes are not produced simultaneously,

(b) at times, the $d B / d t$ rate changes sign in a repetitive mode although the energetic proton flow direction is not influenced.

(c) at local expansions the $d B / d t$ rate increases and the energetic proton fluxes decline; in contrast, the electron fluxes increase, and

(d) occasionally, protons and electrons are accelerated in situ within a stationary TCS. In a case energetic protons are produced before any substorm onset.

\subsubsection{The proposed particle energization mechanism}

Our data support an earlier concept (see, e.g. Sarafopoulos and Sarris, 1988) that a substorm is assembled by putting a large number of distinct elementary activations. Each individual activation center, with $\sim 1$ min duration, is typically made up of its own local thinning and expansion phases. In the context of this work, the thinning phase is tightly associated with tip structure formation and the sketch of Fig. 11 illustrates the successive magnetosphere reconfigurations taking place during an activation. We identify three distinct phases: The pre-tip, tip (local thinning) and post-tip structures. Most importantly, electrons and ions are not accelerated simultaneously. It is observationally inferred that protons (electrons) are mainly accelerated during local thinnings (dipolarizations). The latter requires an interpretation which is exhibited below:

\section{Electron energization}

Given that high energetic electron fluxes are mainly observed on closed magnetic field lines and stronger magnetic fields, we infer, in the first place, that electrons must gain energy in their transverse direction and, therefore, some adiabatic heating takes place. Additionally, at the substorm expansion phase the magnetic field structure convects earthward, the length of the bounce path decreases and the electron energy parallel to the field line increases. Hence, Fermi acceleration must probably be at work. However, if the plasma sheet electron temperature is $\sim 0.5 \mathrm{keV}$, then a percentage of electrons will drastically be accelerated by a yet unspecified mechanism. In the context of this work, our suggestion concerning this mechanism is as follows: We already have assumed that electrons are accumulated-trapped in the tip of an already stretched magnetic field line topology. This negative "electrostatic charge" builds up during the local thinning phase, when the tip formation takes place and the ions are demagnetized. In turn, during the local dipolarization phase a small fraction of the accumulated electrons have the potentiality to 

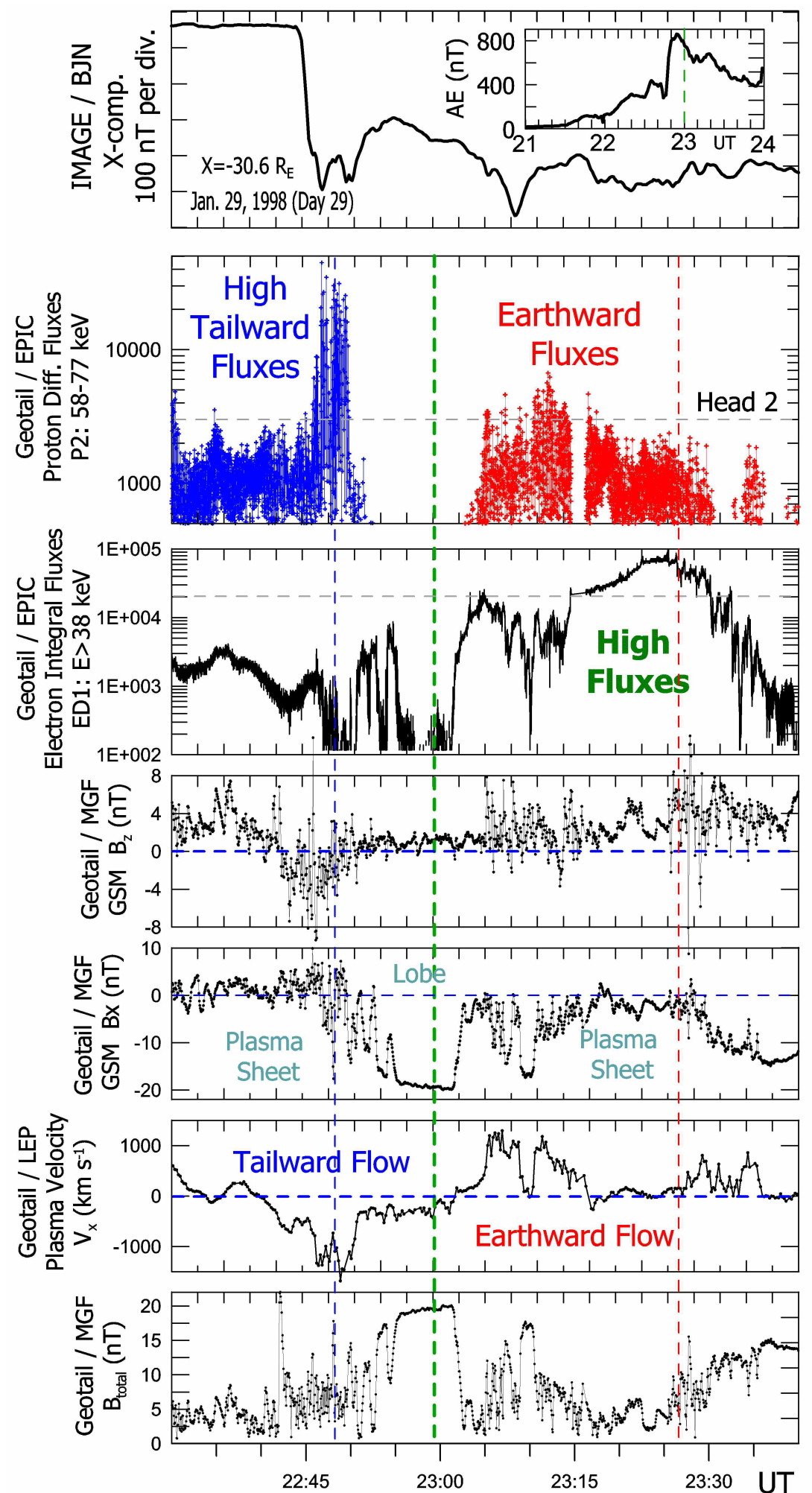

Fig. 10. An isolated moderate substorm on 29 January (day 29), 1994, has been observed by Geotail at $X=-30.6 R_{E}$. Similar format with that of Fig. 9; the ground magnetogram (in nTs) from the BJN station is used in the first panel, while the AE index is shown as an insert for the interval from 21:00 to 24:00 UT. The green-dashed vertical line marks the time on which the plasma changed its flow direction; the blue and red vertical dashed lines mark the highest proton and electron fluxes, respectively. 


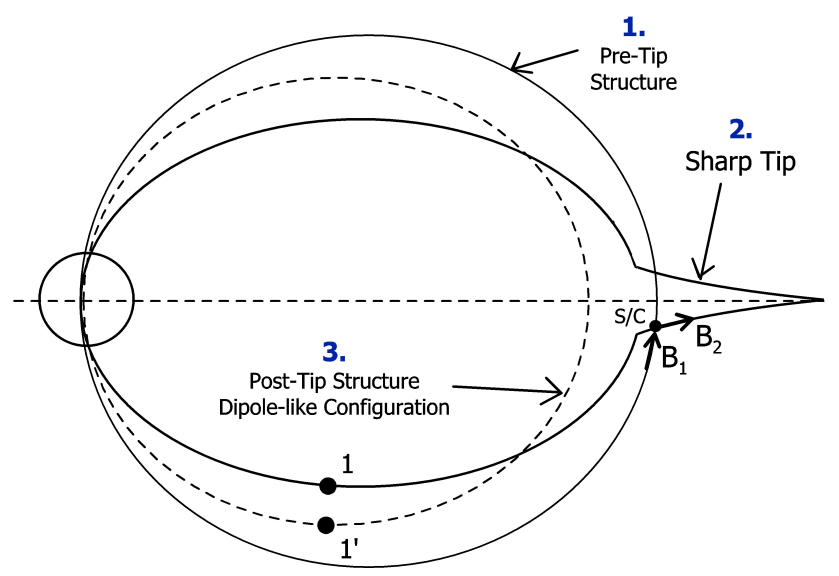

Fig. 11. Schematic illustrating the possible successive magnetic field reconfigurations from the pre-tip (dipole-like structure) to the tip formation, and finally to the post-tip geometry; at higher latitudes the line (1) is moved outward to the position marked as $\left(1^{\prime}\right)$.

escape along the magnetic field lines. The once established electrostatic field has the capacity to accelerate electrons until the bulk charge is eventually degenerated by the repulsion forces among electrons. Figure 12a schematically illustrates the mechanism by which trapped electrons are accelerated.

\section{Proton energization}

At the local thinning phase negative charge is piled up in the tip, and the once established electrostatic field accelerates protons. Figure $12 \mathrm{~b}$ is a sketch showing the mechanism that might produce energetic protons. These protons are expelled (emitted) tailward of the tip, and this picture seems to justify the adopted term "sharp emitter tip".

3.1.3 The proposed acceleration mechanism and the ratio $T_{i} / T_{e} \approx 7$

The ion and electron temperatures in the central plasma sheet show a very high degree of correlation, with $T_{i} / T_{e} \simeq 7$ (for instance see the work of Baumjohann et al., 1989). The linear relation between $T_{i}$ and $T_{e}$ holds for plasma temperatures ranging over a factor of $>\sim 100$, that is, both in a hot and cold plasma sheet and is totally independent of the disturbance level. Therefore, the plasma as well as the energetic ion and electron populations respond collectively as a single unified particle population during plasma sheet temperature transitions (Christon et al., 1988). However, "the heating of ions and electrons in parallel has not been explained yet, either in the framework of the near-Earth neutral line model or by any other theory" (Baker et al., 1996).

We have suggested that the particle acceleration is based on the accumulated negative charge in the tip structure during its formation. That is, under this perspective the acceleration mechanism is based on a "time varying electrostatic electric

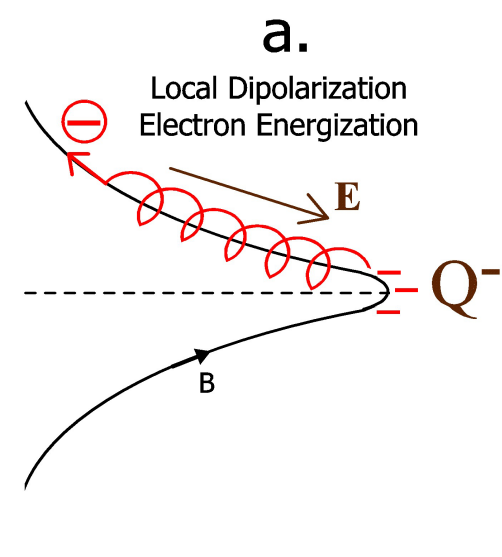

b.

Local Thinning Phase
Proton Energization

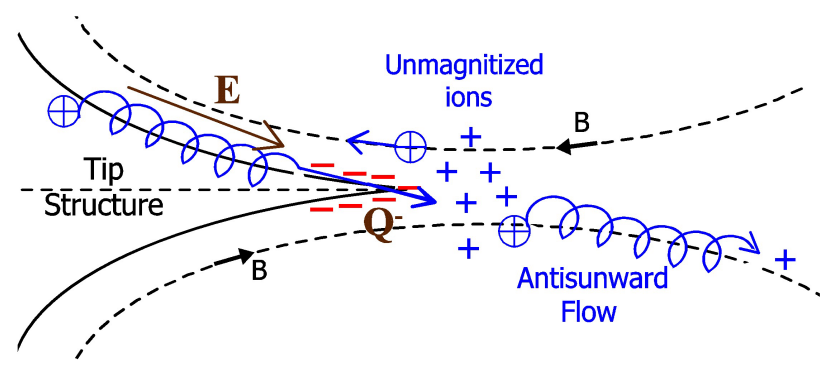

Fig. 12. Mechanism accelerating (a) electrons and (b) protons during local plasma sheet dipolarizations and thinnings, respectively. At the local thinning phase negative charge is piled up in the tip, and the once established electrostatic field accelerates protons. During the local dipolarization phase a small fraction from the accumulated electrons have the chance to undergo acceleration by the same established electrostatic field until the bulk charge is eventually degenerated by the repulsion forces among electrons.

field" along the magnetic field lines. The question is whether such a mechanism might explain the ion to electron temperature ratio.

We can write $T_{i} / T_{e}=m_{i} v_{i}^{2} / m_{e} v_{e}^{2}=7$ or $v_{e} / v_{p} \simeq 16$, where $v_{e}, v_{i}$ and $v_{p}$ are the thermal velocities for electrons, ions and protons, respectively, while the plasma thermal energy is kT. Certainly a simplification is introduced here that all the ions are exclusively protons. Furthermore, we suppose that protons and electrons are accelerated by the same electric field $E$, while their acceleration ratio is $\alpha_{e} / \alpha_{\bar{p}} \widetilde{\sim} 1832$, where $\alpha_{e}$ and $\alpha_{p}$ are the electron and proton accelerations, respectively. Using the equation of kinematics for "constant acceleration" and time flights $t_{p}$ and $t_{e}$ for electrons and protons, respectively, we can write 

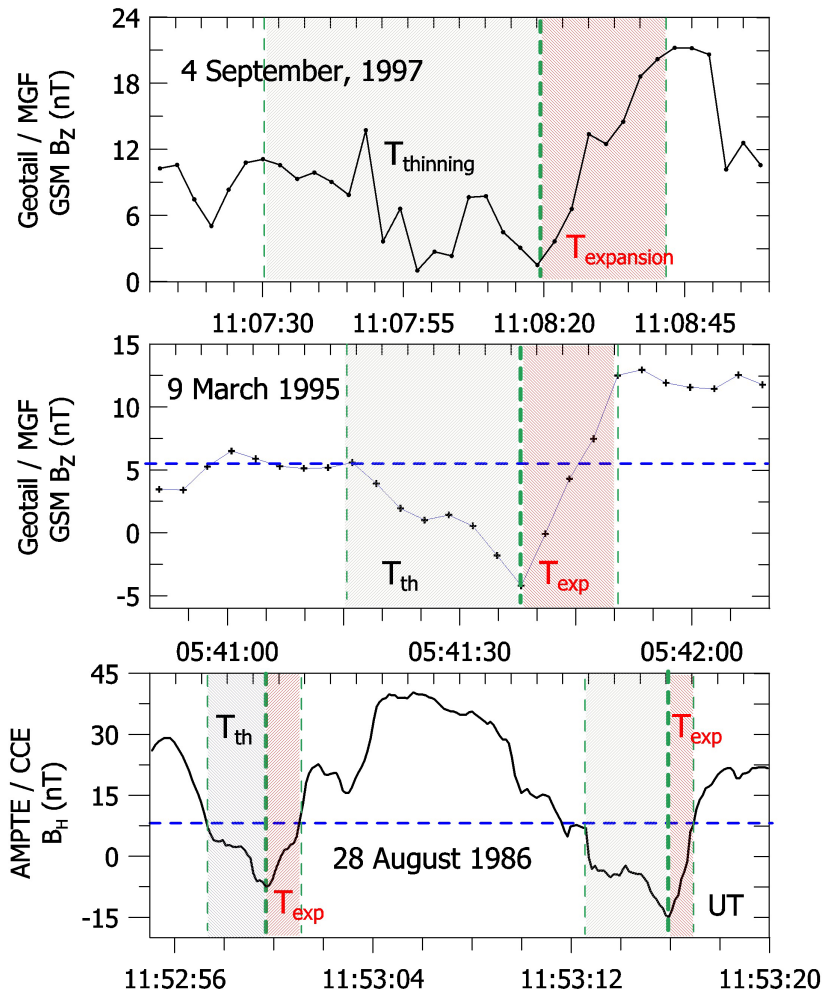

Fig. 13. Four distinct cases corresponding to three different substorms with intense local thinning/expansion phases. The two cases are taken from our presented Geotail data sets of days 247, 1997, and 68, 1995; the third is taken from the well-known substorm of day 240, 1986 (AMPTE/CCE data). The thinning phase identified either by the decreasing $B_{z}$, or by the decreasing north-south component $B_{H}$ of the magnetic field, is apparently 2-4 times longer than the expansion phase.

$\frac{t_{p}}{t_{e}}=\frac{v_{p} / \alpha_{p}}{v_{e} / \alpha_{e}} \simeq 114$

If we hypothesize an ideal electrostatic electric field accelerating particles and having length $\mathrm{L}$, then $\frac{1}{2} \alpha_{e} t_{e}^{2}=\frac{1}{2} \alpha_{p} t_{p}^{2}$, or the same energy is achieved by electrons and protons as well, when

$\frac{t_{p}}{t_{e}}=42$

Therefore, the observational constraint of $T_{i} / T_{p}=7$ leads to $t_{p} / t_{e}=114$, whereas an ideal accelerating source yields $t_{p} / t_{e}=42$. We explain this discrepancy as follows: our basic scheme is that protons are essentially accelerated during local thinnings, whereas electrons are mainly accelerated during local expansions. In both phases the involved "electrostatic charge" is assumed to be the same; the novel point is that the local thinning phase seems to be longer than the expansion phase and, therefore, ions have the opportunity to be accelerated for a longer time. Theoretically, from the preceded simple computation the thinning phase has to be $\sim 2.7$ longer than the expansion one; an anticipation which is observationally supported. In Fig. 13 we show four distinct cases corresponding to three different substorms with intense local thinning/expansion phases. Two cases are taken from our Geotail case studies of days 247, 1997, and 68, 1995; the third is taken from the well-known substorm of day 240, 1986 (AMPTE/CCE data) at a radial distance of $\sim 8 \mathrm{R}_{E}$ near midnight. Actually the thinning phase identified by the decreasing $B_{z}$, or by the decreasing north-south component $\mathrm{B}_{H}$ of the magnetic field in the local coordinate VDH system described by Takahashi et al. (1987), is apparently 2-4 times longer than the expansion phase. The plasma sheet reconfigurations (i.e. thinning and expansion phases) hide in their duration the lifetime of the accelerating mechanism: We postulate that the duration of thinning (expansion) reflects the lifetime $\tau_{\rho}\left(\tau_{e}\right)$ of the proton (electron) accelerating electric field, that is $\tau_{\rho}$ and $\tau_{e}$ are primarily parameters of the accelerating source itself. It must be stressed, however, that we are interested in their ratio, hence $\tau_{p} / \tau_{e} \approx 2.7$. Otherwise, the protons stay at thinnings is 2.7 times longer as compared to their own stay at expansions.

At this point, it is interesting to mention that the above relationship concerning the local thinning to expansion time scale ratio holds, macroscopically, for substorm associated thinnings and recoveries of plasma sheet. Sarafopoulos and Sarris (1990) statistically inferred that the plasma sheet boundary layer duration (i.e. the period spent by the satellite in a region of $10 \%$ depression of the lobe magnetic field) during plasma sheet thinnings is longer than plasma sheet recoveries.

Under the assumptions that

(a) the field-aligned accelerating electric field $E$ is the same for both expansion and thinning phases, since they are coupled through the same charge, and

(b) that there are two separate energization processes for protons and electrons which are associated with $\tau_{p} / \tau_{e} \approx 2.7$,

we infer that the two acceleration sources having lengths $L_{\text {exp }}$ and $L_{t h}$ for the expansion and thinning phases, respectively, are characterized by the ratio

$\frac{L_{t h}}{L_{\exp }}=\frac{2 a_{p} \tau_{p}^{2}}{2 a_{p} \tau_{e}^{2}}=\left[\frac{\tau_{p}}{\tau_{e}}\right]^{2} \simeq 7$

At the expansion phase, although protons and electrons gain the same amount of energy, the electron population is the dominant one, and the achieved energy gain can be written

$\Delta W_{\exp }=q E L_{\exp }$

At the thinning phase protons are mainly energized by $\Delta W_{t h}=q E L_{t h}$. Thus the proton-electron heating process is characterized by $\Delta W_{t h} / \Delta W_{\exp } \simeq 7$.

A transient field-aligned electric field of $100 \mathrm{mV} / \mathrm{m}$ and a scale size $L_{\text {exp }}=1000 \mathrm{~km}$, will produce $100 \mathrm{keV}$ electron and $700 \mathrm{keV}$ proton fluxes. Similar values have been used or estimated in the past (Sarafopoulos and Sarris, 1985; Lopez et al., 1989). 


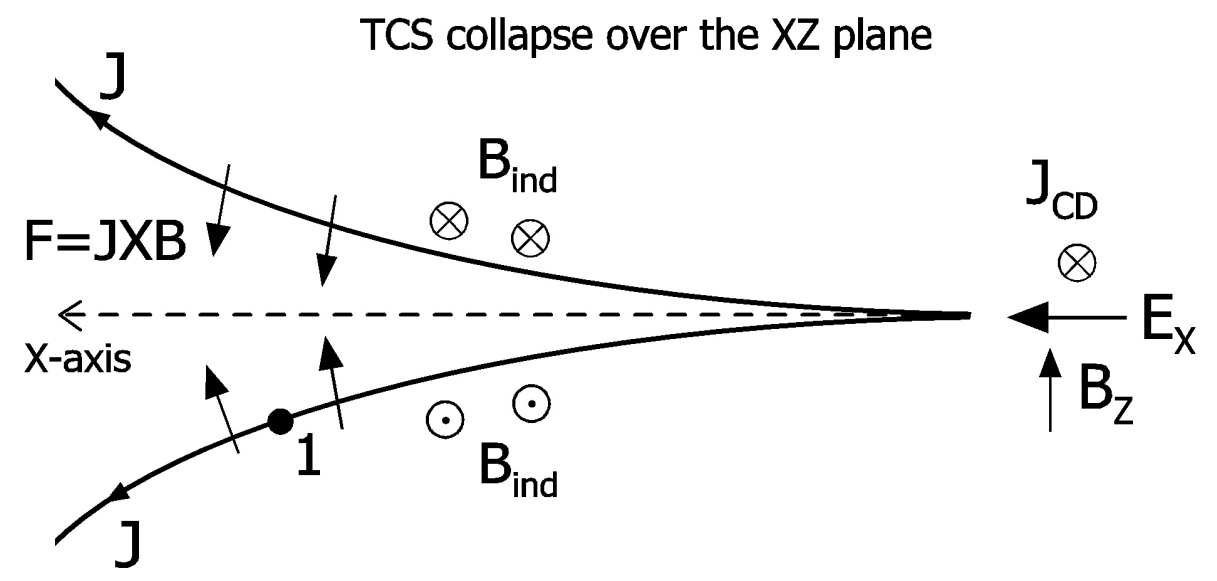

Fig. 14. Schematic over the $X Z$ plane illustrating that the motion of electrons toward the tip could be considered equivalent with two parallel filaments carrying current $J$. The parallel currents are mutually attracted, the filaments move toward each other, the $\bar{J} \times \bar{B}$ force progressively increases and, unavoidably, the TCS structure will collapse. The collapsing tip may develop a strong electrostatic field $E_{x}$ in front of it that points earthward and, potentially, causes current disruption.

To summarize: The magnetized electrons are essentially accelerated on closed topologies during local dipolarizations, while protons attain their high energies during the longer local thinnings. However, the accelerating electric field seems to be the same producing $T_{i} / T_{e} \approx 7$.

\subsection{TCS collapse mechanism}

It is generally accepted that at the substorm growth phase a magnetic field line is highly distorted away from a dipole form by the presence of a thin but intense current sheet (TCS) located in the near-Earth plasma sheet. Under our fundamental assumption, "current-carrying electrons" are piled up and trapped in the tip structure, while the motion of electrons toward the tip could be considered equivalent with two parallel branches-threads carrying current $J$, as it is sketched in Fig. 14. The parallel currents are mutually attracted, the threads move toward each other, the $\bar{J} \times \bar{B}$ force progressively increases and, unavoidably, the TCS structure will collapse. Additionally, the collapse mechanism is probably favoured by another factor: The unmagnetized ions in front of the tip will undergo Speiser orbits across the tail, and the local cross-tail current density will increase. The latter will further reduce the $B_{z}$ component of the tip and speed up its collapse. Moreover, it is reasonable to hypothesize that the collapsing tip develops a strong electrostatic field $E_{x}$ (Fig. 14) in front of it that points earthward. Note that this $E_{x}$ could be roughly estimated by the expression

$E_{x}=q_{l} / 2 \pi \varepsilon_{o} R$

where $q_{l}=Q / L$ is the linear charge density from a total charge $Q$ accumulated in the tip structure and uniformly distributed over a length $L$ along the Y-axis. The $E_{x}$ is estimated in a distance $R$ from the tip, supposed that $R \ll L$. This electric field, at the terminating point of the locally thinned plasma sheet region, naturally stops the ion motion and may lead to the current disruption $\left(J_{C D}\right)$ dictated by the local $E_{x}$ and $B_{z}$ ion drift. Once developed the current $J_{C D}$ may have two major consequences: first, earthward of it probably induces a positive $B_{z}$ component which enforces a local dipole-like geometry and, second, tailward of it probably induces a negative $B_{z}$ component which drastically decreases the pre-existing positive $B_{z}$ and prepares the magnetosphere for the next local thinning phase. The latter will very likely take place in a site slightly displaced tailward as compared to the preceded tip site, and in this way the activation centers seem to be gradually moving tailward. The TCS collapse is followed by the local expansion phase, while the involved field lines are displaced inward at the equator and outwards at high latitudes, to assume a more dipolar form associated with a much reduced tail current. The sketch of Fig. 11 illustrates the possible successive reconfigurations from the pretip (dipole-like structure) to the tip formation, and finally to the post-tip geometry; at higher latitudes the (1) line is moved outward to the position marked as $\left(1^{\prime}\right)$.

\subsection{Ionospheric origin electrons favouring the tip formation and substorm triggering}

Inside the magnetosphere cold-dense plasmaspheric and warm outer ring current electron components are found (see for instance the text book of Baumjohann and Treumann, 1996). The two components are well separated: The hot outer magnetospheric trapped electrons are of solar wind origin and have been energized in the magnetosphere. These fluxes are considerable lower than the low energy plasmaspheric fluxes, indicating the dilute state of the external electron component. The latter is of primary importance for our 
a.

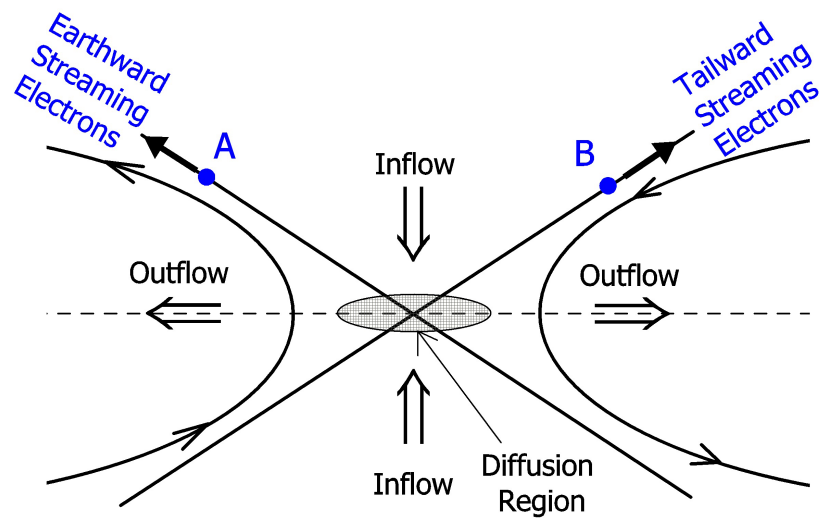

b.

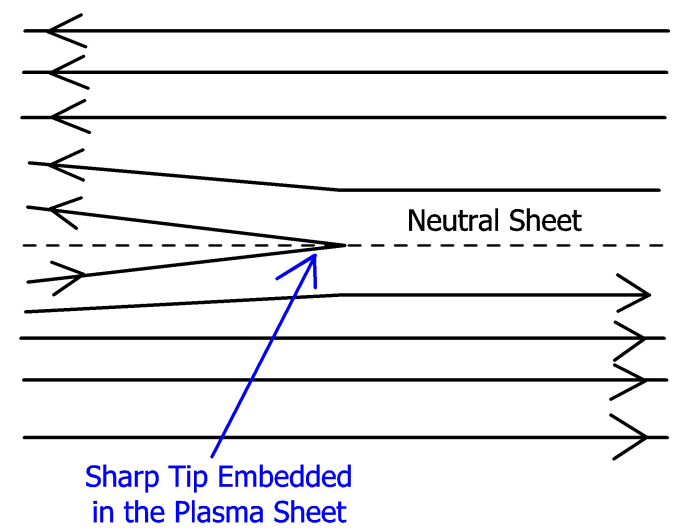

Fig. 15. Schematic illustrating the Petschek's symmetric reconnection model which consists of the inflow region, the outflow region, and the small central diffusion region around a magnetic null situated at the centre of X-line. Beneath it, an asymmetry in the magnetic field topology is introduced which potentially gives an interpretation for the observed particle flux asymmetries in sites like those marked by A and B.

proposed mechanism based on the sharp tip formation and collapse. The dominant cold-dense population of ionosphere electrons with low velocities can readily be trapped in the tip.

We have to pay more attention to the transition region between the ring current and the plasma sheet: The most plasma sheet particles are energetic enough and undergo gradient-drifts encircling the "Alfvén layer" near the Earth. When the $B_{z}$ component of the IMF is strongly negative the magnetosphere convection is strong and the plasma sheet particles can penetrate closer to the Earth, where the plasmaspheric electrons are abundant. The newly built up strong ring current bends the magnetic field lines to a tail-like configuration and the electrons rush up to the tip structure; a TCS may be developed. In turn, an abrupt turning of the IMF to positive $B_{z}$ values will inflate outward the Alfvén layer and the particles, close to the tip, will be trapped on closed- loop trajectories, whereas the plasma sheet particles out of the Alfvén layer will halt penetrating earthward. Therefore, the displaced Alfvén layer is associated with decreased ring current earthward of it and dipolarized magnetic field lines. In this view, a sharp tip is enclosed in a relatively dipole-like structure; an ideal geometry for substorm triggering. Based on this substorm triggering scenario, we see no further obstacles accepting a substorm initiation as close to the Earth as $\sim 8 R_{E}$.

Consequently, the ionospheric electron fluxes seem to play a key role in initiating current disruptions and particle energizations. A similar claim that ionospheric oxygen ions promote substorm activity remains to be demonstrated (Lui, 1996), although it is shown that a general rise in oxygen ion abundance coexists with increased substorm activity (Daglis et al., 1994).

We previously mentioned that an abrupt excursion of the IMF toward positive $B_{z}$ values, after a prolonged interval of southward $B_{z}$, is generally associated with the substorm onset. Similarly, a stepwise dynamic pressure increase in the solar wind may further thin the plasma sheet and lead to the tip formation. In this case, one could anticipate that the near Earth region is first affected and, therefore, the near Earth region may inaugurate the substorm phenomenon.

\subsection{Contradiction in terms of a symmetric reconnection model}

The reconnection process merges magnetic field lines leading to a rearrangement of the magnetic field. In parallel, magnetic field energy is released, heating the plasma, creating a shock wave, and accelerating particles. It is generally accepted that, at least in the distant magnetotail the reconnection takes place on an absolutely symmetric structure, as it is sketched in Fig. 15. That is, the X-line is assumed having a vertical and a horizontal symmetry axis. We show the Petschek's symmetric reconnection model which consists of the inflow region, the outflow region, and the small central diffusion region around a magnetic null situated at the centre of X-line.

It is worth noticing that energetic particles accelerated by reconnection must be of equal fluxes at the plasma sheet boundary layer and at sites symmetric with respect to the $\mathrm{X}$-point. Consequently, satellites positioned at A and B (Fig. 15) have to receive the same amount of earthward and tailward streaming energetic particles, respectively. However, the latter is not observationally supported since a typical asymmetry ratio of 10:1 is observed for the electron fluxes (for instance, in the case of day 44, 1994, Fig. 9). Similar flux asymmetry holds for proton fluxes, distant or near the Earth. Actually, the reconnection is a fundamental concept, but in our view, we have to introduce an asymmetry in the magnetic field topology, which will potentially give an interpretation for the observed particle flux asymmetries. We assume that, even in the distant magnetotail, the sharp tip 


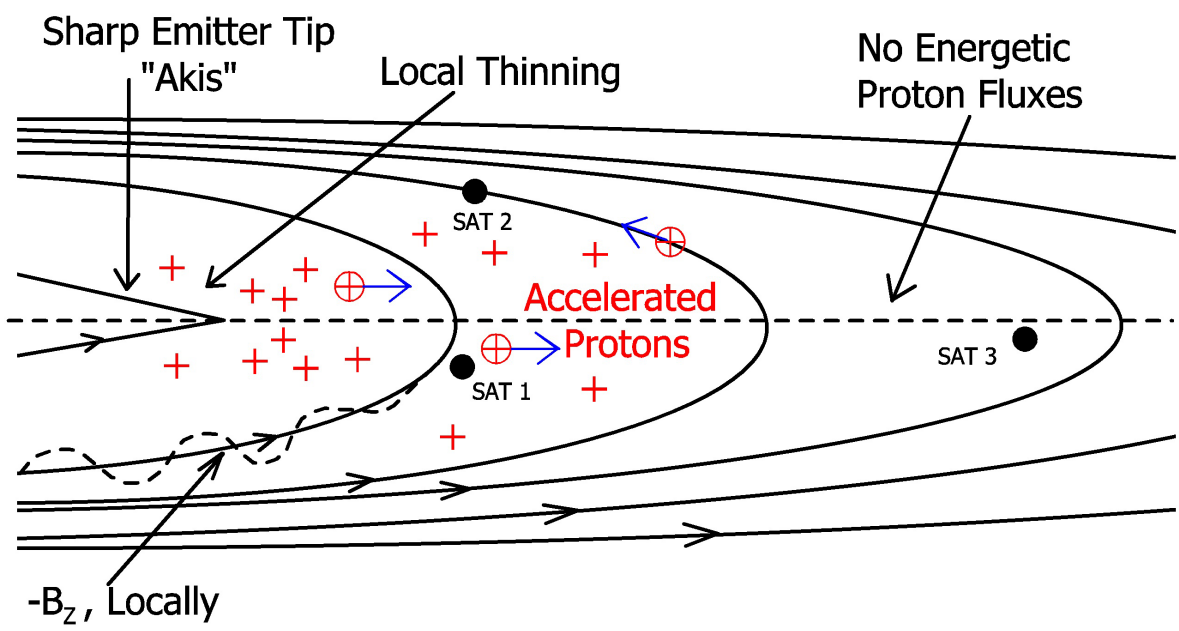

Fig. 16. Once the near Earth tip structure is formed, ions are accelerated toward the tail, but most likely the energized populations are surrounded by closed magnetic field lines. Any ion diffusion process will restrict them to a small range near the Earth, in the tip vicinity. At the SAT 3 site, as it is schematically illustrated, none ion will be detected, while at the SAT 1 (SAT 2) site tailward (earthward) ion fluxes would be detected.

formation plays a vital role. Such a sharp tip embedded in the plasma sheet is schematically shown in Fig. 15, and we believe that this structure is eventually involved in the current disruption-reconnection process. At the substorm recovery phase the acceleration source retreats tailward, and it seems that the sharp tip structure removes tailward as well. In front of the tip the cross tail current may locally disrupt and the anti-parallel magnetic fields lines reconnect. In our reconnection scheme the electrons are essentially kept magnetized earthward of the sharp tip, in front of which a X-line may be formed; however, the dynamics is primarily focused on the knife-edge tip structure.

3.5 Substorm onset site and energetic particle flow direction

The distinguishing signature of the NENL model that tailward flow threaded by southward magnetic field is seldom seen inside of $\sim 20 R_{E}$ (Baumjohann et al., 1990; Angelopoulos et al., 1993) suggests that the $X$ line is typically formed beyond this distance (Baker et al., 1996). In the presented case studies, certainly we have observed tailward fluxes of energetic protons near the Earth $\left(X \cong-10 R_{E}\right)$. Most importantly, we believe that these fluxes may remain undetectable at $X \cong-20 R_{E}$ : once the near Earth tip structure is formed, ions are accelerated toward the tail, but most likely the energized populations are surrounded by closed magnetic field lines. Any ion diffusion process will restrict them to a small range near the Earth, in the tip vicinity. At the SAT 3 site, as it is schematically illustrated in Fig. 16, no ions will be detected, while at the SAT 1 (SAT 2) site tailward (earthward) ion fluxes would be detected. In contrast, highly streaming energetic ion populations could be seen, for instance at $X \cong-180 R_{E}$ (Sarafopoulos et al., 1997), whenever the reconnected field lines reach the separatrix.

\subsection{Sharp emitter tips and the strong-double-layer model}

Following the discussion in the preceding sections, paragraphs a small fraction of the trapped electrons in the tip are accelerated, whereas the bulk population will essentially remain at the initial level of $\sim 0.5 \mathrm{keV}$ temperature. In this subsection, we speculate trying to associate the supposed tip charge with what is called the "strong-double-layer model". It is emphatically stated by Borovsky (1993) that "work is needed to connect auroral-double-layer structures to generator mechanisms in the plasma sheet, in order to extend the model". We assume that the tip formation at local thinnings bunches electrons together, electrons which later at local dipolarizations travel along the magnetic field lines toward their conjugate points in the Northern and Southern Hemispheres. The bottom edge of the double-layer structure resides about $1-2.5 R_{E}$ above the earth's surface, where the ionospheric plasma ions and the magnetospheric plasma electrons have densities that are approximately equal. Much like a parallel-plate capacitor the double-layer may be able to produce the typical auroral-arc-electron energies of $5-15 \mathrm{keV}$. With a plasma sheet temperature of $\sim 0.5 \mathrm{keV}$ the accelerated electrons by the double-layer will be monoenergetic, with the energy being highest near the center of the structure and falling off near the edges.

To summarize: the observations of beam-like distributions of precipitating electrons above auroral arcs led researchers to conclude that there are electrostatic-potential drops above auroral arcs that act to accelerate the electrons downward (e.g. Hoffman and Evans, 1968; Heikkila, 1970; Evans, 


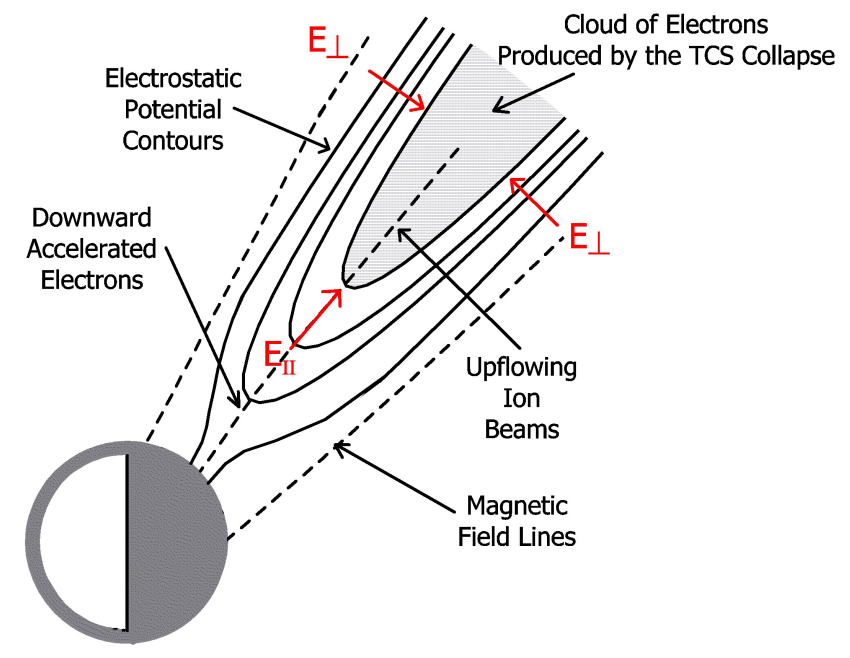

Fig. 17. Schematic illustrating the electrostatic potential drops above auroral arcs that act to accelerate the electrons downward. We suggest that a double-layer generator mechanism might be the "plasma sheet cloud of electrons" originated by the tip structure collapse.

1975; Borovsky, 1992). We suggest that a double-layer generator mechanism might be the locally accumulated negative charge in the sharp tip. Figure 17 is a schematic illustrating the auroral acceleration region established by the "plasma sheet cloud of electrons" originated by the tip collapse.

\subsection{Lack of electron energization at the initial period of a substorm}

The 28 August 1986 substorm-event (Fig. 13) was studied by many researchers; Ohtani et al. (2002) wrote that probably the most puzzling result was that it reveals the lack of electron energization during the initial period of magnetic turbulence. The total magnetic field fluctuated by an order of magnitude during the interval, whereas the electron distribution function seems to be unaffected. This is similar with what we observe the day 247,1997 , from 10:20:30 to 10:23:30 UT (Fig. 3). Energetic protons are abundant, but the electron fluxes are low. In our view, this is the anticipated behaviour, and it constitutes a major observational finding in this work: The energetic proton fluxes do not occur simultaneously with energetic electron fluxes for the reason that energetic protons are mainly accelerated at local thinnings and electrons at local expansions. Moreover, the satellite position is of great importance; generally, proton fluxes and slightly delayed electron fluxes will be observed at positions earthward of the tip, while exclusively proton fluxes will be received tailward of the tip. The schematic Fig. 18 illustrates the geometry; although the various satellite positions (1)-(3) are all characterized by intense $-B_{z}$ at local thinnings, only the position (3) is privileged to be directly associated with the tip structure at the dipolarization phase

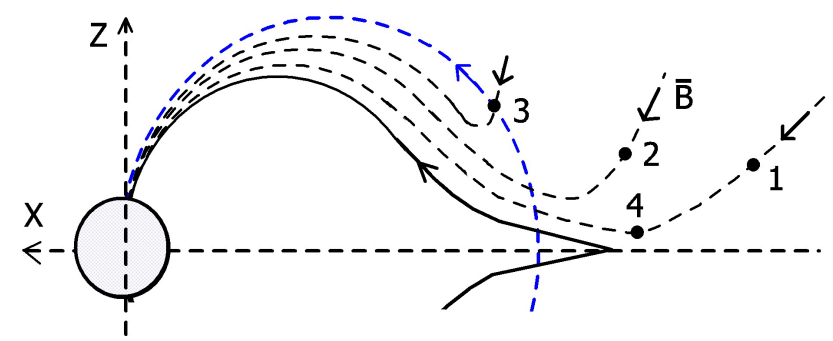

Fig. 18. Schematic illustrating that although the various satellite positions (1)-(3) are all characterized by intense $-\mathrm{B}_{z}$ at local thinnings, only the position (3) is privileged to be directly associated with the tip structure at the dipolarization phase (blue-dashed line). Therefore, newly accelerated electrons can be found only at positions earthward of the tip. In the satellite position (4) only a few demagnetized and tailward streaming energetic electrons can be detected.

(blue-dashed line). Therefore, newly accelerated electrons can be found only at positions earthward of the tip. In the satellite position (4) only a few demagnetized and tailward streaming energetic electrons can be detected; two cases in this category are observed in Figs. 3 and 4. In general, we can state: the longer the interval the satellite senses a dipole structure, the higher the likelihood to detect accelerated electron. The permanent stay in a dipole-like structure is always associated with high electron fluxes supposing that the geomagnetic activity is large enough.

\subsection{The quasi-periodic mode of local dipolarizations}

Takahashi et al. (1987) for the AMPTE/CCE substorm of day 240 (28 August), 1986, detected the quasi-periodic character of $B$ and $B_{H}$ (north-south component) and, among others, stated that a potential physical mechanism exciting these oscillations might be associated with the bounce period of $0.5 \mathrm{keV}$ electrons. In the context of our discussion, such a suggestion may be of great importance; the same periodicity of $T \approx 20$-s, along the $B_{z}$ trace, is detectable in our Figs. 3 and 4. Additionally, we have to underline that the proton bursts typically show longer periodicities, for instance $T \approx 60$-s in our Fig. 1.

Certainly the above mentioned periodicities must be thoroughly studied in the near future in order to support firm results; however, we make a preliminary suggestion. In our context, at the local dipolarization phase the once trapped in the tip structure current-carrying electrons are ejected along the magnetic field lines and prepare the magnetosphere for the next local thinning and dipolarization, in a repetitive fashion. The bounce period of the low energy electrons may determine the time between two successive negative excursions of $B_{z}$; the electron oscillatory flow seems to be in resonance with the successively formed tips. It seems that the bounce period keeps the pace for the growth of repetitive tip structures which loose more and more "demagnetized ions" and, 
in this way, the tip becomes progressively sharper. The increasing number of rebound electrons, which are treated as two parallel currents, may eventually lead to the local TCS collapse (Sect. 3.2), which may be characterized by its own periodicity.

\subsection{Additional comments}

Liu (2001) developed a formulation generating bursty bulk flows (BBFs) without introducing any NENL model. He based on "a highly curved current sheet", which apparently leads to the tip formation, as it is suggested in this work. Additionally, Wanliss et al. (2002) using a test particle model were able to produce fast tailward plasma flows in the near Earth central plasma sheet region as a consequence of plasma sheet boundary layer ion beam dynamics. Certainly they are exclusively focused to the substorm growth phase; however, the same methodology could be progressively applied with a tip structure retreating tailward. The magnetic field model of Wanliss et al. (2000), which take into account an azimuthally and radially confined "weak magnetic field region", is a valuable tool approaching the "tip structure" dynamics. Such an extended model might simulate the proposed acceleration scheme of this work. Eventually, we anticipate that theory and experiment will further converge, in the near future, to diagnose the nature and the role of each substorm related effect.

\section{Summary}

This entire work has, at its core, the introduced "akis" structure. We try to demonstrate its fundamental role in magnetotail dynamics and energetic particle acceleration. The term "akis" involves the local extremely tail-like magnetic field topology and stresses its function as "sharp emitter tip": In the Earth's magnetotail this structure demagnetizes ions and accumulates negative charge. Elsewhere, similar sharp tip structures are proved to work very well (i.e., lightning discharges, Field Emission Electric Propulsion thrusters in satellite technology, Scanning Tunneling Microscopes, etc.), and potentially the same principle may reveal something more of the substorm's mystery.

If the tip formation actually plays such a vital role, and this is further supported by future research, then we could identify the substorm initiating mechanism as the "akis instability". This mechanism may cause current disruption and reconnection as well; however, the driving force is primarily set on the knife-edge tip structure. The observational element that the energetic proton and electron populations are not produced simultaneously is our major finding. We have suggested that the particle energization is essentially gained by transient electrostatic fields along the magnetic field lines, and not by any $d B / d t$ variation according to the traditional assumption. In general, all the long-standing open questions concerning the substorm phenomenon can be approached in a different perspective.

Acknowledgements. Concerning the use of the highest resolution Geotail/EPIC data-sets, we are grateful to (a) A. T. Y. Lui, R. W. McEntire, E. T. Sarris, and D. J. Williams, (b) S. R. Nylund for his pre-processing of the data, and (c) The Johns Hopkins University/Applied Physics Laboratory that maintains the available data web site. We would like to thank Kazue Takahashi for his provision of the 0.124-s resolution AMPTE/CCE magnetic field data of day 240, 1986. Also we thank S. Kokubun and T. Mukai for the high-resolution Geotail/MGF magnetic field and Geotail/LEP plasma data, respectively. Regarding the use of LANL energetic particle data, we thank Richard Belian and Geoffrey D. Reeves. We are grateful to the Finnish Meteorological Institute (FMI/GEO), the Technical University of Braunschweig and other institutes that maintain the IMAGE magnetometer array. The author thanks the three referees who significantly improved the initial manuscript.

Topical Editor I. A. Daglis thanks J. Wanliss and two other anonymous referees for their help in evaluating this paper.

\section{References}

Angelopoulos, V., Kennel., C. F., Coroniti, F. V., Feldman, W. C., Gosling, J. T., Kivelson, M. G., Walker, R. J., and Russell, C. T.: Observations of a quasi-static plasma sheet boundary, Geophys. Res. Lett., 20, 2813-2816, 1993.

Baker, D. N., Pulkkinen, T. I., Angelopoulos, V., Baumjohann, W., and McPherron, R. L.: Neutral line model of substorms: Past results and present view, J. Geophys. Res., 101, 12 975-13010, 1996.

Baumjohann, W., Paschmann, G., and Cattell, C. A.: Average plasma properties in the central plasma sheet, J. Geophys. Res., 94, 6597-6606, 1989.

Baumjohann, W., Paschmann, G., and Lühr, H.: Characteristics of high-speed ion flows in the plasma sheet, J. Geophys. Res., 95, 3801-3809, 1990.

Baumjohann, W. and Treumann R. A.: Basic Space plasma physics, Imperial College Press-ICP, 122-124, 1996.

Belian, R. D., Gisler, G. R., Cayton, T., and Christensen, R.: High$\mathrm{Z}$ energetic particles at geostationary orbit during the great Solar Proton Event Series of October 1989, J. Geophys. Res., 97, 16897-16906, 1992,

Birn, J., Hesse, M., and Schindler, K.: MHD simulations of magnetotail dynamics, J. Geophys. Res., 101, 12 939-12 954, 1996.

Borovsky, J. E.: Double layers do accelerate particles in the auroral zone, Phys. Rev. Lett., 69, 1054-1056, 1992.

Borovsky, J. E.: The Strong-Double-Layer model of auroral arcs: An Assessment, in: Auroral Plasma Dynamics, Geophysical Monogr. Ser., 80, edited by: Lysak, R. L., AGU, 113-120, 1993.

Christon, S. P., Mitchell, D. G., Williams, D. J., Frank, L. A., Huang, C. Y., and Eastman, T. E.: Energy spectra of plasma sheet ions and electrons from $\sim 50 \mathrm{eV} / \mathrm{e}$ to $\sim 1 \mathrm{MeV}$ during plasma temperature transitions, J. Geophys. Res., 93, 2562-2572, 1988.

Christon, S., Williams, D., Mitchell, D., Frank, L., and Huang, C.: Spectral Characteristics of Plasma Sheet Ion and Electron Populations During Undisturbed Geomagnetic Conditions, J. Geophys. Res., 94(A10), 13 409-13 424, 1989. 
Christon, S., Williams, D., Mitchell, D., Huang, C., and Frank, L.: Spectral Characteristics of Plasma Sheet Ion and Electron Populations during Disturbed Geomagnetic Conditions, J. Geophys. Res., 96(A1), 1-22, 1991.

Cowley, S. W. H.: Acceleration and heating of space plasmas: basic concepts, Ann. Geophys., 9, 176-187, 1991, http://www.ann-geophys.net/9/176/1991/.

Daglis, I. A., Livi, S., Sarris, E. T., and Wilken, B.: Energy density of ionospheric and solar wind origin ions in the near-Earth magnetotail during substorms, J. Geophys. Res., 99, 5691-5703, 1994.

Evans, D. S.: Evidence for the low altitude acceleration of auroral particles, in: Physics of Hot Plasma in the Magnetosphere, edited by: Hultqvist, B. and Stenflo, L., Plenum, New York, 1975.

Friedel, R. H. W., Korth, A., and Kremser, G.: Substorm onset observed by CRRES: Determination of energetic particle source regions, J. Geophys. Res., 101, 13 137-13 154, 1996.

Gold, R. E., Bostrom, C. O., and Roelof, E. C.: Anisotropy measurements of $\sim 50 \mathrm{keV}$ solar protons, 14 th Int. Cosmic Ray Conf., Munish, SP 5.2-10, 1975.

Heikkila, W. J.: Are we on the right approach to solve the substorm problem? in Proceedings of the Eighth International Conference on Substorms (ICS-8), edited by Syrjäsuo and Donovan, University of Calgary, Alberta, Canada, 87-92, 2007.

Heikkila, W. J.: Satellite observations of soft particle fluxes in the auroral zone, Nature, 225, 369-370, 1970.

Hoffman, R. A. and Evans, D. S.: Field-aligned electron bursts at high latitudes observed by OGO 4, J. Geophys. Res., 73, 62016214, 1968.

Kokubun, S., Yamamoto, T., Acuna, M. H., Hayashi, K., Shiokawa, K., and Kawano, H.: The GEOTAIL magnetic field experiment, J. Geomagnetism Geoelectricity, 46, 7-21, 1994.

Krimigis, S. M. and Sarris, E. T.: Energetic particle bursts in the Earth's magnetotail, in: Dynamics of the Magnetosphere, edited by: Akasofu, S. I., Reidel Publishing Co., 599-630, 1980.

Liu, W. W.: Bursty bulk flows without a near-Earth neutral line: Generation of fast intermittent flow in a highly curved magnetic field, J. Geophys. Res., 106, 289-300, 2001.

Lopez, R. E., Sibeck, D. G., McEntire, R. W., and Krimigis, S. M.: The energetic ion substorm injection boundary, J. Geophys. Res., 95, 109-117, 1990.

Lopez, R. E. and Baker, D. N.: Evidence for particle acceleration during magnetospheric substorms, The Astrophys. J. supplement series, 90, 531-539, 1994.

Lopez, R. E., Lui, A. T. Y., Sibeck, D. G., Takahashi, K., McEntire, R. W., Zanetti, L. J., and Krimigis, S. M.: On the relationship between the energetic particle flux morphology and the change in the magnetic field magnitude during substorms, J. Geophys. Res., 94, 17 101-17 119, 1989.

Lui, A. T. Y., Chang, C.-L., Mankofsky, A., Wong, H.-K., and Winske, D.: A cross-field current instability for substorm expansions, J. Geophys. Res., 96, 11 389-11 401, 1991.

Lui, A. T. Y.: Current disruption in the Earth's magnetosphere: Observations and models, J. Geophys. Res., 101, 13067-13088, 1996.

Lui, A. T. Y.: Current controversies in magnetospheric physics, Rev. Geophys., 39(4), 535-563, 2001.

Lyons, L. R.: Substorms: Fundamental observational features, distinction from other disturbances, and external triggering, J. Geo- phys. Res., 101, 13 011-13 025, 1996.

Maral, G. and Bousquet, M.: Satellite communications systems, John Wiley and Sons, ltd, fourth edition, 584-586, 2002.

Mukai, T., Machida, S., Satio, Y., Hirahara, M., Terasawa, T., Kaya, N., Obara, T., Ejiri, M., and Nishida, A.: The low energy particle (LEP) experiment onboard the Geotail satellite, J. Geomagnetism Geoelectricity, 46, 669-692, 1994.

Nagai, T., Shinohara, I., Fujimoto, M., Hoshino, M., Saito Y., Machida, S., and Mukai, T.: Geotail observations of the Hall current system: Evidence of magnetic reconnection in the magnetotail, J. Geophys. Res., 106(A11), 25 929-25 949, 2001.

Ohtani, S., Takahashi, K., and Lui, A. T. Y.: Electron dynamics in the current disruption region, J. Geophys. Res., 107, 1322, doi:10.1029/2001JA009236, 2002.

Plonus, M. A.: Applied electromagnetics, McGraw-Hill Book Company, 85-92, 1978.

Rostoker, G.: Phenomenology and physics of magnetospheric substorms, J. Geophys. Res., 101, 12 955-12 973, 1996.

Roux, A., Perraut, S., Morane, A., Robert, P., Korth, A., Kremser, G., Pederson, A., Pellinen, R., and Pu, Z. Y.: Role of the nearearth plasma sheet at substorms, in: Magnetospheric Substorms, edited by: Potemra, T., Geophysical Monograph Series, 64, 201214, 488 pages, hardbound, ISBN 0-87590-030-5, AGU CODE GM0640305, AGU, Washington, D.C., 1991.

Sarafopoulos D. V., Sarris, E. T., and Pavlos, G. P.: Estimate of the amplitude of transient electric fields in the magnetotail, Proceeding Conf. Achievements of the IMS (International Magnetospheric Study), 26-28 June 1984, Graz, Austria, ESA SP-217, 177-180, 1984.

Sarafopoulos, D. V. and Sarris, E. T.: Transient Field-Aligned Electric Fields inside the Plasma Sheet inferred from measurements of Energetic Particles, Planet. Space Sci., 35, 991-995, 1987.

Sarafopoulos, D. V. and Sarris, E. T.: Inverse Velocity Dispersion of Energetic Particle bursts inside the Plasma Sheet, Planet. Space Sci., 36, 1181-1199, 1988.

Sarafopoulos, D. V. and Sarris, E. T.: Substorm Associated Energetic ion $(\mathrm{E} \cong 45 \mathrm{keV})$ Flows at the Plasma Sheet Boundary Layer: A Dawn-Dusk Flow Reversal, Planet. Space Sci., 38, 1251-1266, 1990.

Sarafopoulos, D. V., Sarris, E. T., Angelopoulos, V., Yamamoto, T., and Kokubun, S.: Spatial structure of the plasma sheet boundary layer at distances greater than $180 R_{E}$ as derived from energetic particle measurements on GEOTAIL, Ann. Geophys., 15, 12461256, 1997, http://www.ann-geophys.net/15/1246/1997/.

Sarafopoulos, D. V., Sidiropoulos, N. F., Sarris, E. T., Lutsenko, V., and Kudela, K.: The Dawn-dusk plasma sheet asymmetry of energetic particles: an INTERBALL perspective, J. Geophys. Res., 106, 13 053-13 067, 2001.

Sarris, E. T., Krimigis, S. M., and Armstrong, T. P.: Observations of magnetospheric bursts of high protons and electrons at $\sim 35 R_{E}$ with IMP-7, J. Geophys. Res., 81, 2341-2355, 1976.

Sergeev, V. A., Pulkkinen, T. I., and Pellinen, R. J.: Coupled-mode scenario for the magnetospheric dynamics, J. Geophys. Res., 101, 13047-13065, 1996.

Schindler, K.: A theory of the substorm mechanism, J. Geophys. Res., 79, 2803-2810, 1974.

Takahashi, K., Zanetti, L. J., Lopez, R. E., McEntire, R. W., Potemra, T. A., and Yumoto, K.: Disruption of the magnetotail 
D. V. Sarafopoulos: A physical mechanism producing suprathermal populations

current sheet observed by AMPTE/CCE, Geophys. Res. Lett., 14, 1019-1022, 1987.

Wanliss, J. A., Sydora1, R. D., Rostoker, G., and Rankin, R.: Origin of some anisotropic tailward flows in the plasma sheet, Ann. Geophys., 20, 1559-1575, 2002,

http://www.ann-geophys.net/20/1559/2002/.

Wanliss, J. A., Samson, J. C., and Friedrich, E.: On the use of photometer data to map dynamics of the magnetotail current sheet during substorm growth phase, J. Geophys. Res., 105, $27673-$ $27684,2000$.
Williams, D. J., McEntire, R. W., Schemm II, C., Lui, A. T. Y., Gloeckler, G., Christon, S. P., and Gliem, F.: Geotail energetic particles and ion composition instrument, J. Geomagn. Geoelectr., 46, 39-57, 1994. 